\title{
Midazolam-Resistant Seizures and Brain Injury after Acute Intoxication of Diisopropylfluorophosphate, an Organophosphate Pesticide and Surrogate for Nerve Agents
}

\author{
Xin Wu, Ramkumar Kuruba, and (Doodipala Samba Reddy \\ Department of Neuroscience and Experimental Therapeutics, Texas A\&M University Health Science Center College of Medicine, \\ Bryan, Texas
}

Received December 9, 2017; accepted August 14, 2018

\begin{abstract}
Organophosphates (OP) such as the pesticide diisopropylfluorophosphate (DFP) and the nerve agent sarin are lethal chemicals that induce seizures, status epilepticus (SE), and brain damage. Midazolam, a benzodiazepine modulator of synaptic GABA-A receptors, is currently considered as a new anticonvulsant for nerve agents. Here, we characterized the time course of protective efficacy of midazolam $(0.2-5 \mathrm{mg} / \mathrm{kg}$, i.m.) in rats exposed to DFP, a chemical threat agent and surrogate for nerve agents. Behavioral and electroencephalogram (EEG) seizures were monitored for 24 hours after DFP exposure. The extent of brain injury was determined 3 days after DFP exposure by unbiased stereologic analyses of valid markers of neurodegeneration and neuroinflammation. Seizures were elicited within $\sim 8$ minutes after DFP exposure that progressively developed into persistent SE lasting for hours. DFP exposure resulted in massive neuronal
\end{abstract}

injury or necrosis, neurodegeneration of principal cells and interneurons, and neuroinflammation as evident by extensive activation of microglia and astrocytes in the hippocampus, amygdala, and other brain regions. Midazolam controlled seizures, neurodegeneration, and neuroinflammation when given early (10 minutes) after DFP exposure, but it was less effective when given at 40 minutes or later. Delayed therapy ( $\geq 40$ minutes), a simulation of the practical therapeutic window for first responders or hospital admission, was associated with reduced seizure protection and neuroprotection. These results strongly reaffirm that the DFPinduced seizures and brain damage are progressively resistant to delayed treatment with midazolam, confirming the benzodiazepine refractory SE after OP intoxication. Thus, novel anticonvulsants superior to midazolam or adjunct therapies that enhance its efficacy are needed for effective treatment of refractory SE.

\section{Introduction}

Nerve agents and organophosphate (OP) pesticides are chemical threat agents. Nerve agents are chemical warfare agents that have long attracted the attention of terrorists for attacking a civilian population (Pereira et al., 2014; Reddy and Colman, 2017). Military nerve agents (sarin, soman, tabun, cyclosarin, and VX) directly target the nervous system and rapidly impair neural signaling within minutes of exposure. Sarin is one of the most widely used nerve agents, as evident from recent attacks in Syria and Japan (Yanagisawa et al., 2006; Dolgin, 2013; Rosman et al., 2014). OP pesticidesdiisopropylfluorophosphate (DFP), parathion, and paraoxonare considered credible threat agents (Bouzarth and Himwich, 1952; Baille-Le Crom et al., 1995; Kadriu et al., 2009;

This work was supported by the CounterACT Program, Office of the Director, National Institutes of Health (OD) and the National Institute of Neurologic Disorders and Stroke [Grants U01 NS083460 and R21 NS099009] (to D.S.R.). The authors have no competing financial interests. The views expressed in this paper are those of the author(s) and do not reflect the official policy of the National Institutes of Health or the U.S. government.

https://doi.org/10.1124/jpet.117.247106.
Wright et al., 2010; Liu et al., 2012; Ferchmin et al., 2014; $\mathrm{Li}$ et al., 2015; Flannery et al., 2016). DFP is a potential terrorist threat agent (see Sisó et al. (2017)). In addition, thousands of OP pesticide poisonings occur annually due to suicides or accidents worldwide (Savage et al., 1988; Gunnell and Eddleston, 2003; Jokanović and Kosanović, 2010; Krause et al., 2013). DFP is commonly used as a surrogate for nerve agents to test the efficacy of medical countermeasures in the National Institutes of Health (NIH) CounterACT program in civilian laboratories (Deshpande et al., 2010; Jett and Yeung, 2010; Reddy and Kuruba, 2013; Pessah et al., 2016; Pouliot et al., 2016; Sisó et al., 2017; Liang et al., 2018; Rojas et al., 2018; Scholl et al., 2018).

Nerve agents and OP pesticides are lethal and produce neurotoxicity via common mechanisms (Bajgar, 2004). They primarily cause neurotoxicity due to their irreversible inhibition of acetylcholinesterase, leading to an excessive accumulation of acetylcholine in the synaptic cleft in peripheral and central nervous systems. OPs also bind to inhibitory muscarinic receptors (autoreceptors), which regulate the release of acetylcholine into the synaptic cleft (Bakry et al., 1988;

ABBREVIATIONS: AMN, atropine methylnitrate; BBB, blood-brain barrier; CA, cornu ammonis; DFP, diisopropylfluorophosphate; DG, dentate gyrus; DH, dentate hilus; EEG, electroencephalogram; FJB, fluoro-jade B; GB, [sarin] (RS)-propan-2-yl methylphosphonofluoridate; GD, [soman] 3,3-dimethylbutan-2-yl methylphosphonofluoridate; GFAP, glial fibrillary acidic protein; IBA1, ionized calcium binding adaptor molecule-1; MDZ, midazolam; NeuN, neuronal nuclei; $\mathrm{NIH}$, National Institutes of Health; OP, organophosphate; 2-PAM, pralidoxime; PBS, phosphate-buffered saline; PV, parvalbumin; SE, status epilepticus. 
Pittel et al., 2006, 2018). Acute exposure to OPs results in cholinergic hyperactivation and causes a set of predictable and well-documented toxic signs: hypersecretion, miosis, headache, fasciculations, tremors, convulsions, respiratory distress, and death (Bakry et al., 1988; Bajgar, 1997; McDonough and Shih, 1997; Shih et al., 1991, 2003; Hájek et al., 2004; Bajgar et al., 2008; Sirin et al., 2012; Abou-Donia et al., 2016; Reddy and Colman, 2017; Pittel et al., 2018).

Central nervous system manifestations after OP exposure include convulsive seizures and status epilepticus (SE), which can last 30 minutes or longer causing profound brain damage that results in neuronal damage or death (Chen, 2012; Hobson et al., 2018; Scholl et al., 2018). Brain damage is thought to occur not only by seizure-related excitotoxicity (Shih et al., 2003; Prager et al., 2013) but also via mechanisms independent of seizures such as activation of glia and cellular inflammation (Yokoyama, 2007; Banks and Lein, 2012; Pereira et al., 2014). The effects of OP intoxication are long lasting, and survivors suffer chronic brain damage including the risk of neurologic and cognitive deficits (Shih et al., 2003; Yokoyama, 2007; Liu et al., 2012; Prager et al., 2013; Li et al., 2015; Rojas et al., 2015; Flannery et al., 2016; Sisó et al., 2017).

The current standard of care for OP intoxication includes: 1) atropine, a muscarinic receptor antagonist, 2) pralidoxime (2-PAM), an acetylcholinesterase reactivator, and 3) diazepam, a benzodiazepine anticonvulsant (McDonough and Shih, 1997; Bajgar, 2004; Eddleston et al., 2008; Shih et al., 2009; McDonough et al., 2010; Reddy, 2014, 2016). Benzodiazepines are the first-line anticonvulsants for nerve agent seizures, but they have important limitations. Diazepam must be given within 30 minutes, after which there is reduced protection against seizures, and progressive neuronal damage occurs (Hayward et al., 1990; Lallement et al., 1997; McDonough and Shih, 1997; Shih et al., 1999; Capacio et al., 2001; Goodkin et al., 2003; Skovira et al., 2010; Apland et al., 2014; Rogin et al., 2014; Reddy, 2016). This time line is often not practical in scenarios such as after a chemical attack. Moreover, diazepam autoinjector formulation has suboptimal pharmacokinetics, and repeated doses of diazepam are needed to control recurrent seizures, resulting in sedation, respiratory depression, and tolerance in victims (Hassel, 2006; Goodkin and Kapur, 2009; McDonough et al., 2010; Rogin et al., 2014). After OP exposure, it is critical to control seizures at an early stage for neuroprotection, survival, and preventing brain dysfunction (Shih et al., 2003).
Midazolam (MDZ) has been proposed as a replacement anticonvulsant to diazepam for OP intoxication (Gilbert et al., 1999; Reddy and Reddy, 2015; Glauser et al., 2016; Smith and Brown, 2017). MDZ is an effective anticonvulsant in experimental paradigms (McDonough et al., 1999, 2009; Shih et al., 1999; Koplovitz et al., 2001; Capacio et al., 2004; RamaRao et al., 2014). The RAMPART (Rapid Anticonvulsant Medication Prior to Arrival Trial, a NIH-sponsored clinical trial) study showed that MDZ can control SE in outpatient settings (Silbergleit et al., 2011, 2012). However, there are many reports with evidence of seizure recurrence or resistance after an initial protection by MDZ and especially with delayed postexposure treatment after OP intoxication (McDonough and Shih, 1997; McDonough et al., 1999; Kwong, 2002; Leikin et al., 2002; Chapman et al., 2015; Reddy and Reddy, 2015).

In this study, we characterized the time course of protective efficacy of MDZ in the DFP model of OP intoxication-induced SE and brain injury in rats. Our results indicate that DFP-induced SE and brain injury are progressively resistant to delayed postexposure (40 minutes or later) MDZ therapy, confirming the benzodiazepine refractory SE after OP intoxication.

\section{Material and Methods}

Animals. Adult male Sprague-Dawley rats (250-300 g) (Taconic Farms, Rockville, MD) were used in the study. The rats were maintained in animal facility under a 12-hour light/dark cycle with ad libitum access to food and water. All procedures were completed under a protocol approved by the university's institutional animal care and use committee in compliance with the guidelines of NIH Guide for the Care and Use of Laboratory Animals.

Experimental Approach. The study was designed to evaluate the efficacy of MDZ as an anticonvulsant antidote for OP intoxication. The overall experimental protocol is illustrated in Fig. 1. We rigorously tested the efficacy of $\mathrm{MDZ}$ in a well-characterized model of acute OP intoxication, the DFP rat model (oral $\mathrm{LD}_{50}=5 \mathrm{mg} / \mathrm{kg}$ ). The actual study design was intended to evaluate both the time course and dose-response relationships of MDZ to efficacy.

Rats were randomly assigned to groups using the randomization sequence generation. Table 1 summarizes the experimental design and group size. The sample size $(\mathrm{N})$ needed for each experiment was calculated using the power analysis for obtaining statistically significant ( $\alpha=0.05$ ) outcomes based on Lamorte's power calculations, on the magnitude of effect observed and its variability in our preliminary studies, and on published reports (Briyal and Reddy, 2008; Apland et al., 2010; Figueiredo et al., 2011; Kuruba and Reddy, 2011).

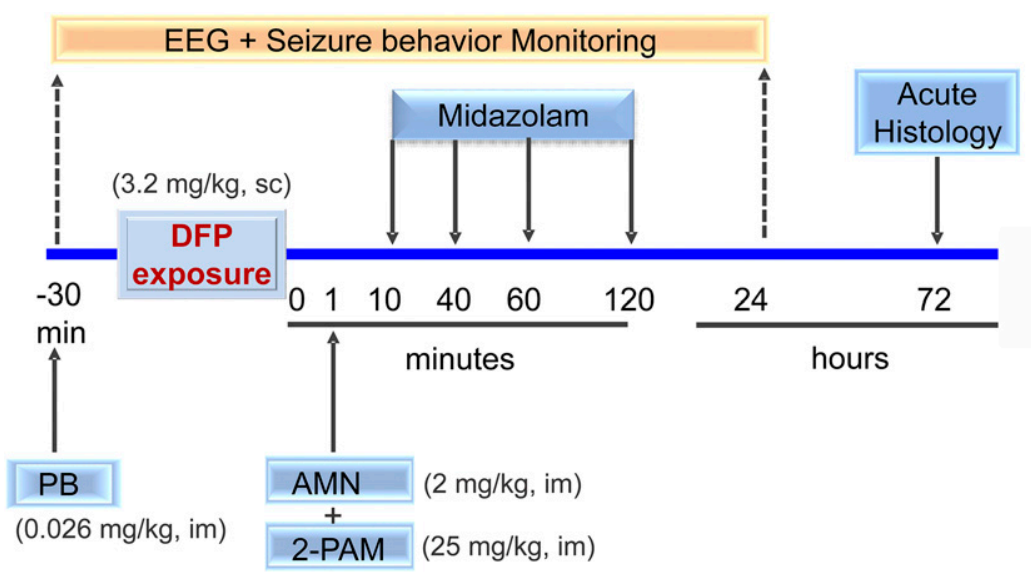

Fig. 1. Experimental paradigm for evaluating anticonvulsant efficacy of midazolam in the DFP model of organophosphate intoxication. Timeline " 0 " is the time of chemical exposure. Rats were pretreated with pyridostigmine bromide (PB, $0.026 \mathrm{mg} / \mathrm{kg}$, i.m.) 30 minutes before DFP exposure. Rats were given a standard antidote regimen AMN and 2-PAM after DFP (3.2 mg/kg, s.c.). Midazolam $(2 \mathrm{mg} / \mathrm{kg}$, i.m. $)$ was given at $10,40,60$, or 120 minutes after DFP exposure. Rats were monitored for behavioral and EEG seizures for 24 hours and then perfused at 72 hours for brain histology (see Material and Methods for details). 
Time-course profile of midazolam (2 mg/kg, i.m.) in the DFP model in rats

\begin{tabular}{|c|c|c|c|c|c|}
\hline \multirow{3}{*}{ Response } & \multicolumn{5}{|c|}{ Number (\%) Rats Showing Response } \\
\hline & \multirow{2}{*}{$\frac{\text { Vehicle }}{10 \min (n=8)}$} & \multicolumn{4}{|c|}{ Time Point for Midazolam Administration after DFP } \\
\hline & & $10 \min (n=8)$ & $40 \min (n=15)$ & $60 \min (n=15)$ & $120 \min (n=14)$ \\
\hline SE termination & $0(0 \%)$ & $8(100 \%)$ & $3(21 \%)$ & $1(7 \%)$ & $0(0 \%)$ \\
\hline Latency for SE termination & NT & $\sim 10 \mathrm{~min}$ & NT & NT & NT \\
\hline 24-Hour mortality $(\%)$ & $5 / 10(50 \%)$ & $0 / 8(0 \%)^{a}$ & $3 / 15(20 \%)^{a}$ & $5 / 15(33 \%)$ & $5 / 14(36 \%)$ \\
\hline
\end{tabular}

DFP, diisopropylfluorophosphate; NT, not terminated within 2-hour period; SE, status epilepticus.

${ }^{a} P<0.05$ versus vehicle control (Wilcoxon test).

The power and sample size were computed based on the proposed statistical tests including one-way and two-way repeated measures analysis of variance for neuropathologic results.

A sample size of $8(N=8)$ was found to be adequate for the timecourse study of MDZ in the DFP seizure model (Table 1). A sample size of four or more was found to be sufficient for stereologic analysis of neuroprotection outcomes. Test drugs were evaluated in a dose-dependent and time-course fashion (see Table 1). For efficacy determination, MDZ was given by an intramuscular (i.m.) injection 10 minutes (early therapy), 40 minutes (critical period, i.e., a simulation of practical therapeutic window for first responders and emergency care), and 1 or 2 hours after OP exposure (late therapy) because previous studies showed refractoriness to diazepam at these time points (Goodkin et al., 2003; McDonough et al., 2010; Apland et al., 2014). Behavioral and electroencephalogram (EEG) seizures were recorded continuously for 24 hours. Acute histologic outcome was assessed at 72 hours after DFP exposure. Appropriate controls were used, including: 1) vehicletreated rats as controls for the test drug, and 2) electrode-implanted rats as an additional control group.

Experimental Outcomes and Analysis. We analyzed three outcomes of MDZ treatment effectiveness: 1) anticonvulsant efficacy, 2) neuroprotectant efficacy, and 3) prevention of neuroinflammation. The anticonvulsant efficacy of MDZ in terminating DFP-induced SE and seizure activity was assessed using four parameters: 1) severity of behavioral seizures, 2) frequency of electrographic spikes, 3) cumulative duration of seizure activity, and 4) latency to termination of seizure activity. EEG data were analyzed by Mini-analysis and Origin software. SE was considered terminated when the EEG returned to normal baseline or showed irregular spikes of $<1$ spike/s. Behavioral seizures were considered terminated when stage $<2$ and there was normal behavior or inactivity.

The neuroprotectant efficacy was assessed by an immunohistochemical staining and quantitative analysis of brain sections using three specific markers: 1) neuronal injury (fluoro-jade B; FJB), 2) principal neuronal loss (neuronal nuclei; NeuN), and 3) interneuron loss (parvalbumin; PV) in key brain regions including the hippocampus, amygdala, thalamus, entorhinal cortex, somatosensory cortex, and piriform cortex. The antineuroinflammation efficacy was assessed by immunohistochemically using two specific markers: 1) astrocyte activation/injury (glial fibrillary acidic protein; GFAP), and 2) microglia activation/injury (ionized calcium binding adaptor molecule-1; IBA1) in the hippocampus subfields and other key regions.

EEG Electrode Implantation. Rats were anesthetized with an intraperitoneal (i.p.) injection of a mixture of ketamine $(100 \mathrm{mg} / \mathrm{kg})$ and xylazine $(10 \mathrm{mg} / \mathrm{kg})$. Rats were fixed in the stereotaxic system (David Kopf Instruments, Tujunga, CA). Stainless steel EEG recording electrodes (PlasticsOne, Roanoke, VA) were implanted on the surface of the brain over the right frontoparietal cortex and over the left cerebellum as a reference electrode. An intracranial depth electrode was placed into the right dentate gyrus as per the following coordinates with reference to the bregma: $-4 \mathrm{~mm}$ anteroposterior, $2.3 \mathrm{~mm}$ mediolateral, and $3.5 \mathrm{~mm}$ dorsoventral. Rats were allowed to acclimate for 1 to 2 weeks to recover from the surgery.
DFP Model of OP Intoxication. DFP was purchased from Sigma-Aldrich (St. Louis, MO) and stored at $4^{\circ} \mathrm{C}$. Dilutions were made freshly with ice-cold phosphate-buffered saline (PBS). We adapted a DFP protocol that was well established within the NIH CounterACT program (Deshpande et al., 2010; Reddy and Kuruba, 2013; Pouliot et al., 2016; Heiss et al., 2016). DFP (3.2 mg/kg, subcutaneously (s.c.)) was administered to induce persistent seizures and SE in rats. Animals were pretreated with pyridostigmine bromide $(0.026 \mathrm{mg} / \mathrm{kg}$, i.m. $) 30$ minutes before DFP injection. One minute after DFP injection, rats were given pralidoxime chloride $(2-\mathrm{PAM}, 25 \mathrm{mg} / \mathrm{kg}$, i.m.) and atropine methylnitrate (AMN, $2 \mathrm{mg} / \mathrm{kg}$, i.m.) to increase the survival rates without affecting the severity of seizures because AMN and 2-PAM did not significantly cross the blood-brain barrier (BBB) (Kenley et al., 1982; Boccia et al., 2003; Sakurada et al., 2003; Shih et al., 2010). This regimen is consistent with protocols published by the U.S. Army Medical Research Institute of Chemical Defense (McDonough and Shih, 1997; McDonough et al., 1999, 2000; Hobson et al., 2017; Scholl et al., 2018).

The DFP dose was selected based on the $\mathrm{LD}_{50}$ in rats and preliminary experiments conducted in accordance with previous publications (Deshpande et al., 2010; Kadriu et al., 2011; Li et al., 2011; Sisó et al., 2017). Moreover, this dosage induced consistent seizure activity and $\mathrm{SE}$ in adult rats, a key criterion of an animal model for testing anticonvulsants for OP intoxication. DFP is very similar in structure to the nerve agents soman and sarin, so it is widely accepted as a surrogate for nerve agents.

$\mathrm{AMN}$ and 2-PAM have poor BBB penetration. AMN is a bulky charged molecule with inferior ability to penetrate the brain (Boccia et al., 2003). 2-PAM is a charged pyridinium compound with a quaternary ammonium ion, so it cannot effectively cross the BBB (Kenley et al., 1982; Sakurada et al., 2003; Shih et al., 2010). They both act in the peripheral nervous system and are lifesaving antidotes for maintaining respiration and other vital functions.

Video EEG and Behavior Recording. Behavior and EEG activity was monitored continuously for 24 hours after DFP to assess the progression of seizure activity and SE after DFP. A 1-hour baseline EEG recording was conducted before each experiment by using a digital EEG system (Grass-Astromed, Warwick, RI). Rats were awake and freely moving in cages fitted with swivels. DFP-induced EEG seizure activity was observed with the appearance of high amplitude (at least twice the amplitude of the baseline wave) and repetitive discharges $(>0.5 \mathrm{~Hz})$. The severity of behavioral seizures were rated according to the Racine scale (Racine, 1972): stage 0, normal behavioral activity; stage 1 , chewing and facial twitches; stage 2 , head nodding or shaking; stage 3, unilateral forelimb clonus without rearing, straub tail, and extended body posture; stage 4, bilateral forelimb clonus plus rearing; and stage 5 , wild jumping and tonicclonic activity.

Midazolam Treatment. The experimental design for drug administration and outcome measurements is summarized in Fig. 1. MDZ was tested in a time-course and dose-response design. In the time-course study, animals were randomized into six groups $(n=6-15$ per group): control (no DFP), DFP, DFP + MDZ (10 minutes), DFP + MDZ (40 minutes), DFP + MDZ (60 minutes), and DFP + MDZ 
(120 minutes). This design used $2 \mathrm{mg} / \mathrm{kg} \mathrm{MDZ,} \mathrm{which} \mathrm{is} \mathrm{considered} \mathrm{a}$ human equivalent dose for MDZ as an anticonvulsant for SE (Lee et al., 2011; Fernandez et al., 2014; Glauser et al., 2016; Smith and Brown, 2017).

In the dose-response study, animals were randomized into seven groups ( $n=6-15$ per group): control (no DFP), DFP, and DFP + MDZ $(0.2,1,2,3$ or $5 \mathrm{mg} / \mathrm{kg}, 40$ minutes after DFP). The control group received a vehicle injection in a volume equivalent to the drug-exposure groups.

Brain Fixation and Histology Processing. Rats were humanely euthanized or perfused at 72 hours after DFP exposure. In brain perfusion experiments, rats were anesthetized with ketamine $(100 \mathrm{mg} / \mathrm{kg})$ and xylazine $(10 \mathrm{mg} / \mathrm{kg})$ and perfused with heparinized saline (10 units heparin $/ \mathrm{ml}$ saline) followed by $4 \%$ paraformaldehyde solution in PBS (pH 7.4). For the best perfusion, the rat body was fixed with its head down $\left(45^{\circ}\right.$ angle) and perfused transcardially with a pump speed of $35 \mathrm{rpm} / \mathrm{min}$. First, it was perfused with $150 \mathrm{ml}$ of saline mixed with $1 \mathrm{ml}$ of heparin. Then, heparinized saline solution was replaced with $4 \%$ paraformaldehyde $(300 \mathrm{ml})$, and the perfusion was continued. The brains were excised and postfixed in $4 \%$ paraformaldehyde overnight at $4^{\circ} \mathrm{C}$ and then stored in PBS for another 24 hours.

They were processed for cryoprotection serially with $10 \%$ sucrose (48 hours), $20 \%$ sucrose (48 hours), and $30 \%$ sucrose (48 hours). Then brain samples were stored in $30 \%$ sucrose in $0.1 \mathrm{M}$ phosphate buffer (pH 7.4) for 72 hours, rapidly frozen in precooled isopentane $\left(-70^{\circ} \mathrm{C}\right)$, and stored at $-80^{\circ} \mathrm{C}$ until sectioning.

Serial coronal $(30 \mu \mathrm{m})$ sections were cut through the forebrain containing the hippocampus and amygdala relative to the bregma $-0.24 \mathrm{~mm}$ to $-7.44 \mathrm{~mm}$ (Paxinos and Watson, 2007). The sections were collected serially in a 24 -well plate with cold PBS. Every 20 th section (600 $\mu \mathrm{m}$ intervals) through the entire hippocampus was then selected from each rat (total 10 sections per animal; $n=4-11$ rats per group) and processed for immunohistochemistry (Golub et al., 2015). All sections were stored free-floating in section storage solution at $-20^{\circ} \mathrm{C}$ before further processing.

Immunostaining of Fluoro-Jade B (FJB+) Injured Neurons. To evaluate the effect of MDZ on DFP-induced neuronal injury or the number of dying neurons, brain sections were processed for $\mathrm{FJB}(+)$ immunostaining as described previously elsewhere (Schmued et al., 1997; Rao et al., 2006). Briefly, after an overnight air-dry at $22^{\circ} \mathrm{C}$, slides with brain sections were treated sequentially with the following solutions: $100 \%$ ethyl alcohol (3 minutes), $70 \%$ ethyl alcohol (1 minute), distilled water ( 1 minute), $0.06 \%$ potassium permanganate (10 minutes), distilled water (1 minute), and $0.004 \%$ FJB (Histo-Chem, Jefferson, $\mathrm{AR}$ ) in $0.1 \%$ acetic acid (30 minutes) in the dark with slow shaking. Then, the brain sections were rinsed with distilled water twice, air dried for 4 hours in the dark, dehydrated in an ascending alcohol concentration, cleared in xylene, and cover slipped with distyrene plasticizer xylene.

$\mathrm{FJB}(+)$ counting of neurons in the hippocampus subfields was conducted by unbiased stereology (Golub et al., 2015). Evaluation of $\mathrm{FJB}(+)$ neurons in extrahippocampal regions, including the thalamus, hypothalamus, amygdala, piriform cortex, somatosensory cortex, and entorhinal cortex, followed the neuropathology score rating system (Myhrer et al., 2005; Apland et al., 2010). Briefly, the tracings from the Nissl-stained sections were superimposed on the FJB-stained sections. Scores were based on the severity of FJB(+) staining of neurons and loss of neurons in other stainings: $0=$ no neuropathology (0\% staining); 1 = minimal neuropathology ( $1 \%-10 \%$ staining); $2=$ mild neuropathology (11\%-25\% staining); $3=$ moderate neuropathology (26\%-45\% staining); and $4=$ severe neuropathology ( $>45 \%$ staining). Such assessment has been previously shown to produce results that are in agreement with quantitative stereology assessments (Qashu et al., 2010).

Immunohistochemistry of Principal Neurons, Interneurons, and Cellular Neuroinflammation. The extent of neurodegeneration and neuroinflammation in the brain sections was analyzed as per the methodology and protocols published elsewhere (Rao et al., 2006; Kuruba et al., 2011; Golub et al., 2015). Brain sections through the hippocampus were randomly selected in each group for various key markers of neuronal loss, neurodegeneration, and neuroinflammation. Principal neurons and cells were identified and analyzed by $\mathrm{NeuN}(+)$ immunostaining of brain sections (Mullen et al., 1992). GABAergic interneurons were identified and analyzed by $\mathrm{PV}(+)$ immunostaining. The extent of cellular neuroinflammation in the brain was assessed by immunostaining of brain sections for GFAP $(+)$, a validated marker of astrocytosis, and IBA1(+), a commonly used marker for microglia.

After preparation, slides were incubated overnight at room temperature in mouse anti-NeuN antibody (1:1000 in PBS; Chemicon, Temecula, CA), mouse anti-PV antibody (1:2000 in PBS; SigmaAldrich), rabbit anti-GFAP antibody (1:1000 in PBS; Dako North America, Carpinteria, CA), or rabbit anti-IBA1 polyclonal antibodies (1:2000 in PBS; Wako Chemicals, Richmond, VA). Biotinylated secondary anti-mouse IgG solution (1:200; Vector Laboratories, Burlingame, CA) was used with the avidin-biotin complex reagent (Vector Laboratories) for detection (Hattiangady et al., 2011; Kuruba et al., 2011). Diaminobenzidine was used as a chromogen for peroxidase reaction (Vector Laboratories).

The brain sections were mounted on slides, dehydrated in alcohol, cleared in xylene, and coverslipped with DPX Mountant (SigmaAldrich). The extent of neurodegeneration was quantitated in different regions such as cornu ammonis (CA1, CA2, and CA3), dentate gyrus (DG), and dentate hilus (DH) of the hippocampus 3 days after SE. Quantification of cell numbers was performed on one randomly selected side of the brain slices due to the overall neurodegeneration mostly appearing symmetrical in the brain (Rao et al., 2006; Kuruba et al., 2011; Golub et al., 2015). The absolute number of immunopositive neurons (NeuN, PV, or FJB) in different subfields of the hippocampus in multiple groups were counted by unbiased stereology via optical fractionator probe in the Visiopharm's stereology system (Golub et al., 2015).

Neurostereology Protocol. The total number of neurons, relative percent neuroprotection, cell density, and tissue volume in the previously mentioned stained sections were quantified by a validated stereology protocol published elsewhere (Golub et al., 2015). The stereology system consists of newCAST software (version VIS 4.0; Visiopharm, Hørsholm, Denmark) and Olympus BX53 microscope (Olympus, Tokyo, Japan) mounted with a color camera (DP73, Model: DP73-1-51; Olympus) and CCD camera (ORCA-R2; Hamamatsu, Hamamatsu City, Japan). A detailed protocol for design-based stereologic quantification and analysis of neuronal injury and neurodegeneration, including the number of brain sections and number of fields per brain section used for calculating the aggregate cell counts, is described in an article by Golub et al. (2015).

Subjective bias was minimized by blinded analysis of the histology sections and blinded compilation of the stereology data by the counters. The number of surviving neurons were quantified at $5 \%$ of total region area for $\mathrm{NeuN}(+), \mathrm{PV}(+)$, and $\mathrm{FJB}(+)$ cells at magnification $60 \times$ in the CA1, CA3, and DG subfields. The total area was increased to $10 \%$ for $\mathrm{NeuN}(+), \mathrm{PV}(+)$, and $\mathrm{FJB}(+)$ counts in the CA2 and $\mathrm{DH}$ subfields. The total area selection was based on the relative density cells in different brain regions to ensure optimal sampling for stereologic analysis of cell counts. The volume estimation of specific subfields was made with at least 200 points at $10 \times$ objective lens (Boyce et al., 2010; Dorph-Petersen and Lewis, 2011; Golub et al., 2015).

Area Fractionation Densitometry. For quantification of neuroinflammation, the extent of activation of $\operatorname{GFAP}(+)$ astrocytes and IBA1(+) microglial responses was assessed by area fraction densitometry using NIH ImageJ software (Shetty et al., 2004). For each subfield, one image for each slice (at least five images for each rat) was taken in the same anatomic position at the coordinates relative to bregma (e.g., $-3.00,-3.60,-4.20,-4.80$, and $-5.40 \mathrm{~mm}$ at anteroposterior axis), under $20 \times$ objective lens. 
For measurements of area fraction of $\mathrm{GFAP}(+)$ or IBA1 $(+)$ cells in ImageJ, each image was converted to 16-bit grayscale. A threshold value was selected to keep all the GFAP $(+)$ or IBA1(+) expression in brain sections, but not the background area (Shetty et al., 2004). The area occupied by the cell structures was then evaluated by selecting the "Analyze Particles" component in the ImageJ using identical particle parameters. This procedure estimated the area fraction of particles to the total area of the cells examined.

Test Drugs. Sterile MDZ injection $(5 \mathrm{mg} / \mathrm{ml})$ was purchased from Akorn (Lake Forest, IL). DFP, atropine, and 2-PAM were purchased from Sigma-Aldrich. Ketamine, xylazine, heparin, and dextrose sterile injection solutions were procured from Patterson Veterinary (Houston, TX). Midazolam was diluted in sterile saline. DFP was diluted in cold sterile PBS solution. Drug solutions were administered intramuscularly, intraperitoneally, or subcutaneously in a volume less than $0.1 \%$ of the animal's body weight $(0.1 \mathrm{ml} / 100 \mathrm{~g})$ as appropriate for the route of injection. All other reagents in this study were purchased from Sigma-Aldrich unless otherwise noted.

Statistical Analysis. Statistical tests were performed using SAS software (SAS Institute Inc., Cary, NC) and Macrocal Origin 8 (OriginLab Corporation, Northampton, MA). In all statistical tests, differences were considered statistically significant at $P<0.05$. Data were expressed as the mean \pm S.E.M. Sample sizes in various groups are indicated in Table 1 and the figure legends.

First, the data were subjected to a normality test to stratify outcomes into parametric (follows a normal distribution) and nonparametric (does not follow a normal distribution) category. We used the Shapiro-Wilk method to check the normality. Second, the parametric data were subjected to a homogeneity of variance Levene's test before numerous statistical analyses. Statistical comparisons of seizure activity and neuroprotection outcomes were performed with one-way or two-way repeated measures analysis of variance as appropriate. Post hoc analyses were performed to identify specific differences using Tukey's honestly significant difference for multiple comparisons. Nonparametric outcomes, such as mortality rate, were compared between groups using the Wilcoxon signed rank-test. Behavior seizure score and neuropathology score outcomes were analyzed with the nonparametric Kruskal-Wallis test followed by the Mann-Whitney $U$ test.

A correlation analysis was performed to determine the relationship between two independent variables, such as the strength of the association between EEG seizure activity and behavioral seizure severity or neuronal damage and seizure severity. The main neuropathological outcome was the extent of neuronal damage in stained sections across multiple regions of the brain (hippocampus, amygdala, thalamus, frontal cortex, and piriform cortex) over time with MDZ therapy, for each rat in four different cohorts of DFP-exposed rats. Time of MDZ therapy after DFP exposure was treated as a continuous variable in the model (fixed effect) to capture the pattern of neuronal damage severity over treatment time. Multiple neuropathologic indices per animal from the time course of drug therapy were included in linear mixed-effects regression analysis with time for MDZ treatment as a continuous predictor and a random effect for animal to evaluate extent of neuronal protection across regions (Stroup, 2012). These models included brain region and MDZ treatment time and the interactions between regions as a within-animal repeated measure and time of MDZ therapy. The estimated Spearman correlation coefficients and corresponding $95 \%$ confidence intervals were calculated to assess the significance of interactions between neuroprotection and time for MDZ treatment.

\section{Results}

Effect of Midazolam on DFP-Induced Acute Seizures and SE. To characterize the efficacy of MDZ as a postexposure anticonvulsant, we tested it at several time points. Early therapy is defined as MDZ administration at 10 minutes after exposure. Late therapy is defined as administration at either 40, 60, or 120 minutes after DFP intoxication (Fig. 1). Exposure of rats to DFP triggered rapid cholinergic crisis, which was characterized by excessive salivation, body twitches, and behavioral signs of chewing and head nodding (stage 2). These events quickly progressed into SE with clonic seizures (stage 3), and finally generalized tonic-clonic seizure activity with rearing and falling (stage 5) at around 8-10 minutes after DFP exposure. The 24-hour survival rate was $\sim 50 \%$ after DFP without any anticonvulsant treatment.

The survival rate for the groups treated with $\mathrm{MDZ}(2 \mathrm{mg} / \mathrm{kg})$ is listed in Table 1. A higher survival rate was evident in rats treated with $\mathrm{MDZ}(2 \mathrm{mg} / \mathrm{kg})$ at 10 minutes $(100 \%)$ and 40 minutes (80\%) after DFP exposure. The EEG recordings from the hippocampus showed the progression of DFP-induced SE-like spiking activity (Fig. 2A). The spikes began 5-10 minutes after DFP exposure with a frequency of $0.5-15 \mathrm{~Hz}$ (Fig. 2A). The SE was very intense and persistent for many hours after DFP (Fig. 2, A and B). Differences in seizure control among the MDZ-treated groups at various time points are outlined in Fig. 2, B and D, and Table 1.

Administration of MDZ (2 mg/kg, i.m.) at 10 minutes after DFP exposure resulted in a rapid termination of electrographic SE activity (Fig. 2B) and behavioral seizures (Fig. 2C). However, there was a rebound effect as seizures returned within 2 hours (Fig. 2, A and B), indicating that while MDZ administered at 10 minutes after DFP exposure quickly terminates electrographic seizures, it does not completely prevent recurrence of electrographic seizures between 2 and 6 hours after DFP exposure. MDZ administered at 40,60, or 120 minutes after DFP exposure showed temporary reduction in intensity of both behavioral and electrographic SE, or had little effect as evident from continued seizures or seizure recurrence within 30-40 minutes (Fig. 2, A-C).

These time-course results suggested that MDZ (2 mg/kg) was relatively less effective at preventing seizures when administered at 40 minutes or later after DFP exposure relative to MDZ 10-minute group. The vehicle has no effect on seizures activity.

EEG alterations were temporally correlated with behavioral seizure manifestations in both the DFP alone without MDZ treatment and MDZ (40-minute) treatment groups (Fig. 2D). The correlation between behavioral seizure score and EEG activity was statistically significant $(P<0.05)$ during a 4-hour recording after DFP exposure in both DFP alone without MDZ and MDZ groups (Fig. 2D). The correlation between EEG spiking levels and seizure severity was stronger during the incidences of generalized seizures, with more intense activity (stage 4 to 5) compared with the incidences of clonic seizures with less intense activity (stage 2 to 3 ), although both were significant (Fig. 2E).

Using 40 minutes as the critical therapeutic window, we determined the dose-dependent effect of MDZ $(0.2-5 \mathrm{mg} / \mathrm{kg}$, i.m.) on DFP-induced SE at 40 minutes after DFP exposure. Higher doses of MDZ provided better protection against seizures (Fig. 3A), but the survival rate remained unchanged at doses $2 \mathrm{mg} / \mathrm{kg}$ or higher (Fig. 3B). There was maximal protection at $\geq 2 \mathrm{mg} / \mathrm{kg}$ of $\mathrm{MDZ}$ with a survival rate of $80 \%$ (Fig. 3B).

Overall, MDZ treatment effectively terminated seizures when given early (10 minutes) after DFP exposure and moderately reduced seizures at 40 minutes after DFP exposure. 
A

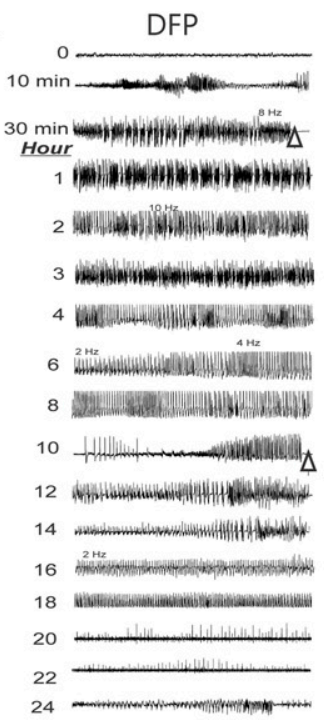

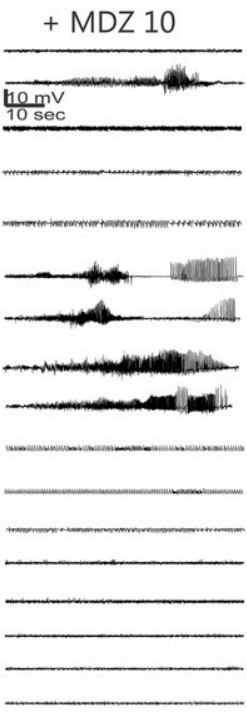
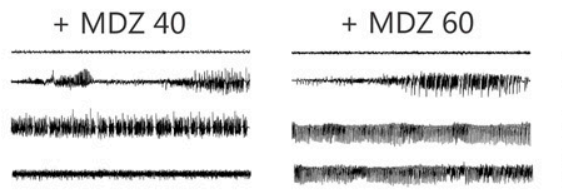

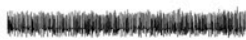
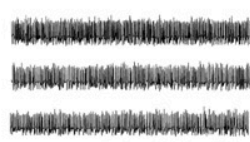

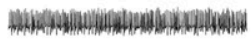
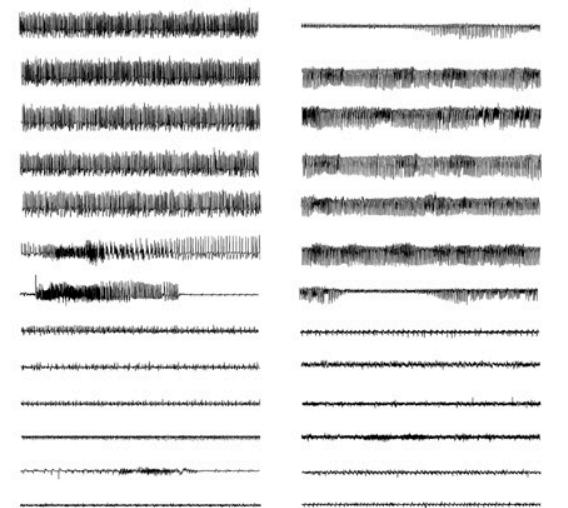

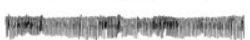
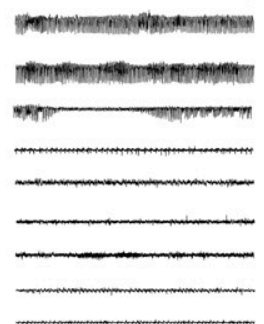
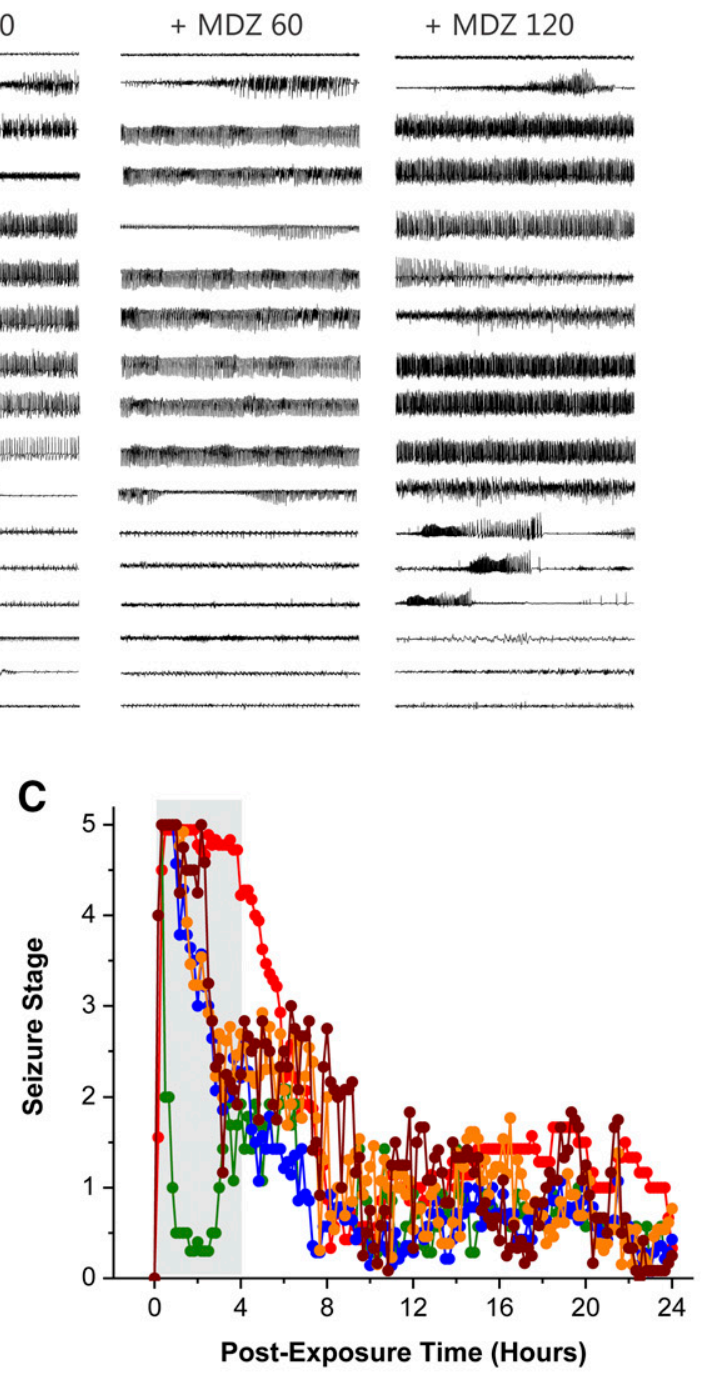

E

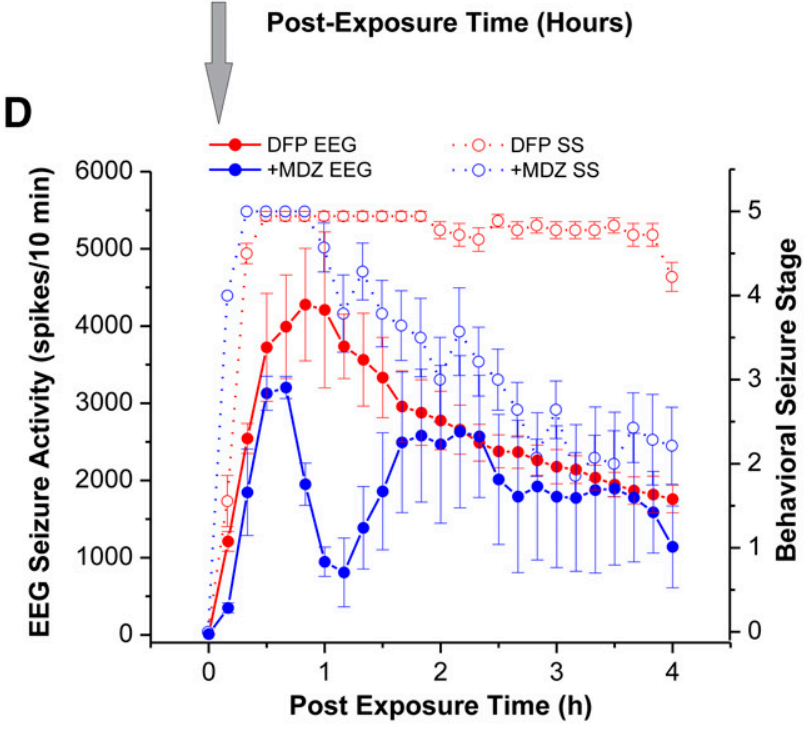

B

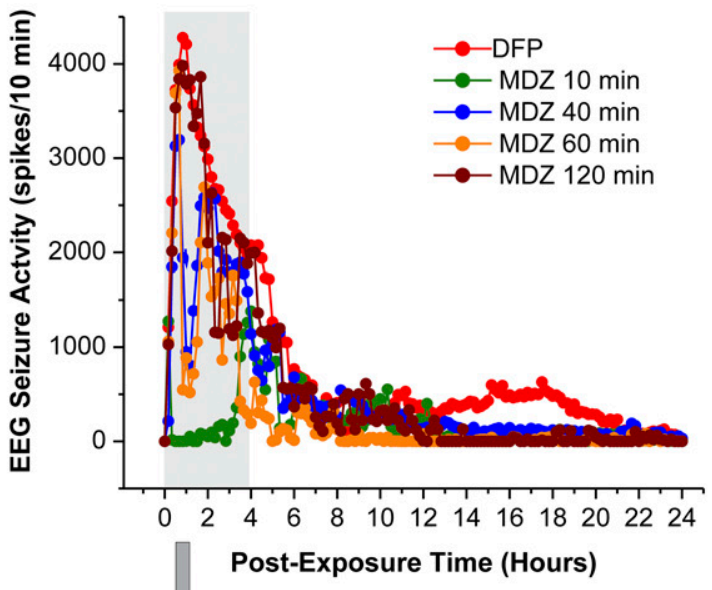

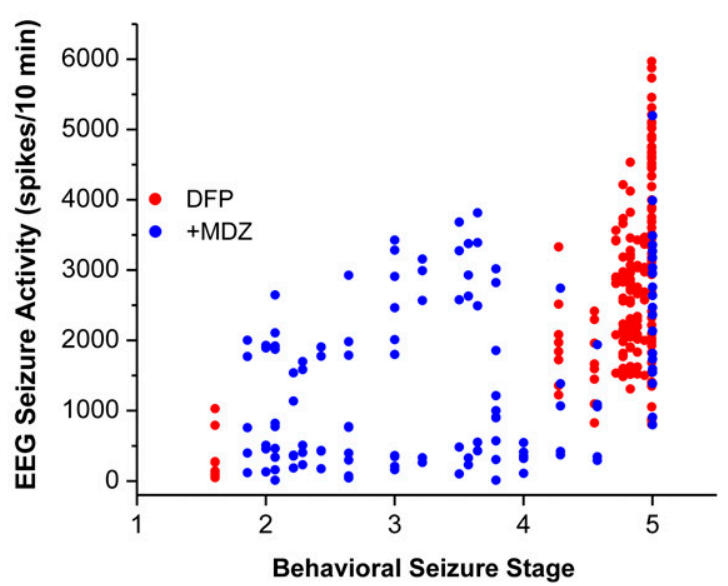

Fig. 2. Time-course profile of midazolam (MDZ, $2 \mathrm{mg} / \mathrm{kg}$, i.m.) on behavioral seizures and EEG seizure activity in the DFP model in rats. (A) Traces represent 1-minute epochs from a depth electrode in the hippocampus. Seizures began $\sim 8-10$ minutes after DFP exposure with intermittent bursts of spikes that progressed into a persistent high amplitude spikes (frequency: $0.5-15 \mathrm{~Hz}$ ). (B) Time-course of EEG seizure suppression by midazolam treatment at 10-120 minutes after DFP exposure. (C) Time-course of behavioral seizure suppression by midazolam treatment at 10-120 minutes after DFP exposure. Each point represents the mean response at 5- or 10-minute intervals (from each group). (D) Temporal correlation between EEG seizure activity and behavioral seizure score in DFP alone and MDZ 40-minute groups. Solid lines indicate the average EEG spike activity after administration of midazolam. Dotted lines indicate the behavioral seizure severity, measured as per the Racine scale. Values represent the mean \pm S.E.M. (from each group). (E) Scatterplot depicting the relationship between the EEG seizure activity and behavioral seizure score during 4 hours after exposure to DFP in DFP alone and MDZ 40-minute groups. Each symbol represents the data from individual animal. The relationship between increase in EEG spiking and 

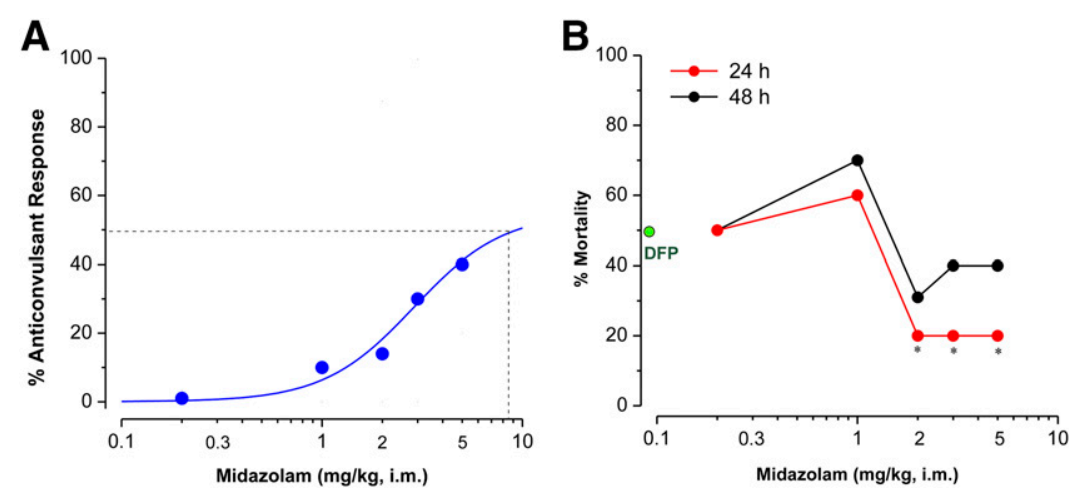

Fig. 3. Dose-response profile of midazolam on seizure activity and survival in the DFP model in rats. (A) Sigmoid plot of midazolam protection against DFP-induced seizures. Midazolam $(0.2-5 \mathrm{mg} / \mathrm{kg}$, i.m.) was given 40 minutes after DFP exposure. Responders were determined by termination or reduction of seizure activity within $60 \mathrm{~min}$ utes after drug injection. (B) Mortality rate in midazolamtreated groups at 24 and 48 hours after DFP exposure. Each point represents the group incidence data $(n=8-15$ rats per group). $* P<0.05$ versus DFP alone (green dot) group (Wilcoxon signed-rank test).
However, it was largely ineffective to control SE at 60 or 120 minutes after DFP exposure, indicating the reduced seizure protection offered by delayed MDZ treatment.

Effect of Midazolam on DFP-Induced Acute Neuronal Injury and Necrosis in the Hippocampus and Amygdala. The neuroprotective effect of MDZ was tested 3 days after exposure to DFP. The extent of neuronal injury or dying neurons was examined by $\mathrm{FJB}(+)$ staining of brain sections and stereologic quantification of injured neurons in the hippocampus subfields CA1, CA2, CA3, and DG as well as the amygdala. In FJB-stained sections, the injured neurons exhibited a bright green fluorescence in DFP groups (Fig. 4A).

The number of $\mathrm{FJB}(+)$ cells significantly increased in the hippocampus and amygdala in the DFP-exposed group (Fig. 4B). Normalized neuronal protection in the groups that received MDZ at 10, 40, 60, and 120 minutes after DFP exposure was determined based on comparisons with the untreated, DFPexposed group (Fig. 4C). MDZ ( $2 \mathrm{mg} / \mathrm{kg}$ ) administered 10 minutes after DFP exposure significantly reduced DFP-induced neuron loss (70\%-90\% protection) in the hippocampus subfields and amygdala (Fig. 4B). A significant reduction in absolute $\mathrm{FJB}(+)$ cell counts was evident in the hippocampus (total and DG subfield), but not in the amygdala, in animals that received MDZ at 40 minutes, and in some cases at even later time points, relative to DFP alone (Fig. 4B). Nevertheless, total FJB(+) cell counts remained significantly higher in these groups compared with MDZ administered at 10 minutes after DFP exposure (Fig. 4, B and C).

Moreover, there was significantly reduced neuroprotection in most brain regions examined in brain sections from MDZ 40-, 60-, and 120-minute subgroups as evident from the percentage of neuroprotection (Fig. 4C) and FJB(+) cell density (Fig. 4D) relative to control and rats that received $\mathrm{MDZ}$ at 10 minutes after DFP exposure. The volume for hippocampus $\left(74.5 \pm 0.3 \mathrm{~mm}^{3}\right.$ in control) and its subfields of CA1, CA2, CA3, DG, and DH, as well as amygdala $\left(22.4 \pm 0.7 \mathrm{~mm}^{3}\right)$, were not significantly different between the control and DFP-exposed groups (Fig. 4E), indicating little change in tissue volume/size at 3 days after DFP exposure.

Overall, these neuropathologic results suggest that early (10 minutes), but not late (40 minutes or after), administration of MDZ is associated with significant protection against DFP-induced neuronal injury and necrosis in the brain.

Effect of Midazolam on DFP-Induced Acute Neuronal Injury and Necrosis in Extrahippocampal Brain Regions. The DFP-induced neuronal injury was determined in other extrahippocampal brain regions, including thalamus, hypothalamus, amygdala, piriform cortex, somatosensory cortex, and entorhinal cortex (Fig. 5A). Given the tedious nature of stereology counts for numerous regions, we used a simple neuropathology-based relative quantification of neuronal damage in these regions using a neuropathology scoring of 1 (low) to 4 (high).

As shown in Fig. 5B, after DFP exposure, extensive neuronal injury was evident in the thalamus, piriform cortex, amygdala, somatosensory cortex, hypothalamus and entorhinal cortex in DFP alone group. The hypothalamus had the lowest $\mathrm{FJB}(+)$ cells compared with the other brain regions (Fig. 5B). The extent of neuronal injury was significantly lower in animals treated with MDZ at 10 minutes after DFP (Fig. 5, B and C).

However, there was limited or reduced protection in other treatment groups when MDZ was given at 40 minutes or later after DFP when compared with DFP alone and rats that received MDZ at 10 minutes after DFP exposure (Fig. 5, B and C). Thus, these morphologic results suggest that early MDZ treatment (10 minutes) provides greater protection against DFP-induced neuronal injury in extrahippocampal regions, which is consistent with the limited neuroprotection outcomes in the hippocampus and amygdala. Moreover, even though the scoring method is suboptimal to stereology-based quantification of cell loss (Fig. 4B), the percentage of neuronal injury assessed by neuropathology scoring system (Fig. 5C) was somewhat consistent with quantitative unbiased stereology counts in the amygdala region (Fig. 4C).

Effect of Midazolam on DFP-Induced Acute Degeneration of NeuN(+) Principal Neurons. To evaluate whether MDZ therapy is associated with protection of principal neurons in the brain, we evaluated brain sections using $\mathrm{NeuN}(+)$ immunostaining at 72 hours after DFP exposure (Fig. 6). The $\mathrm{NeuN}(+)$ staining and stereology counting of control rat hippocampus sections resulted in a total of 1.4 million neurons in the hippocampus (Fig. 6B). Massive

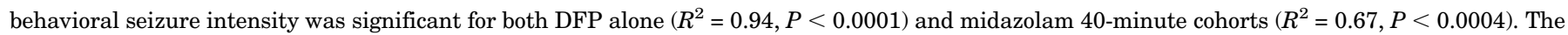

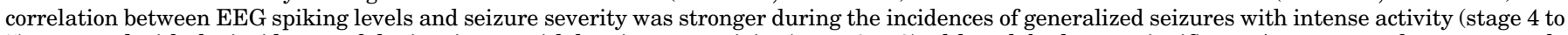

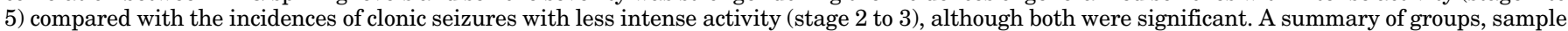
size, and outcomes is listed in Table 1. 
A

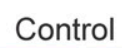

DFP
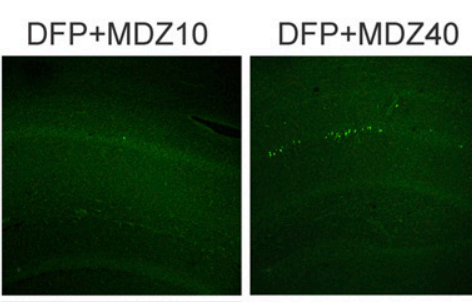

DFP+MDZ120
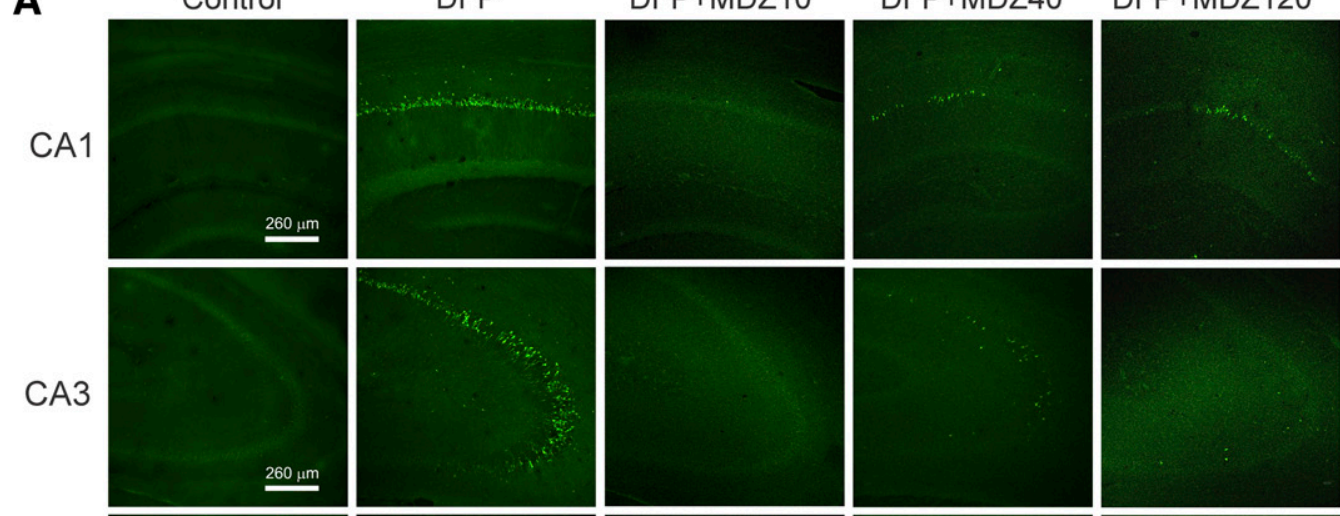

DG
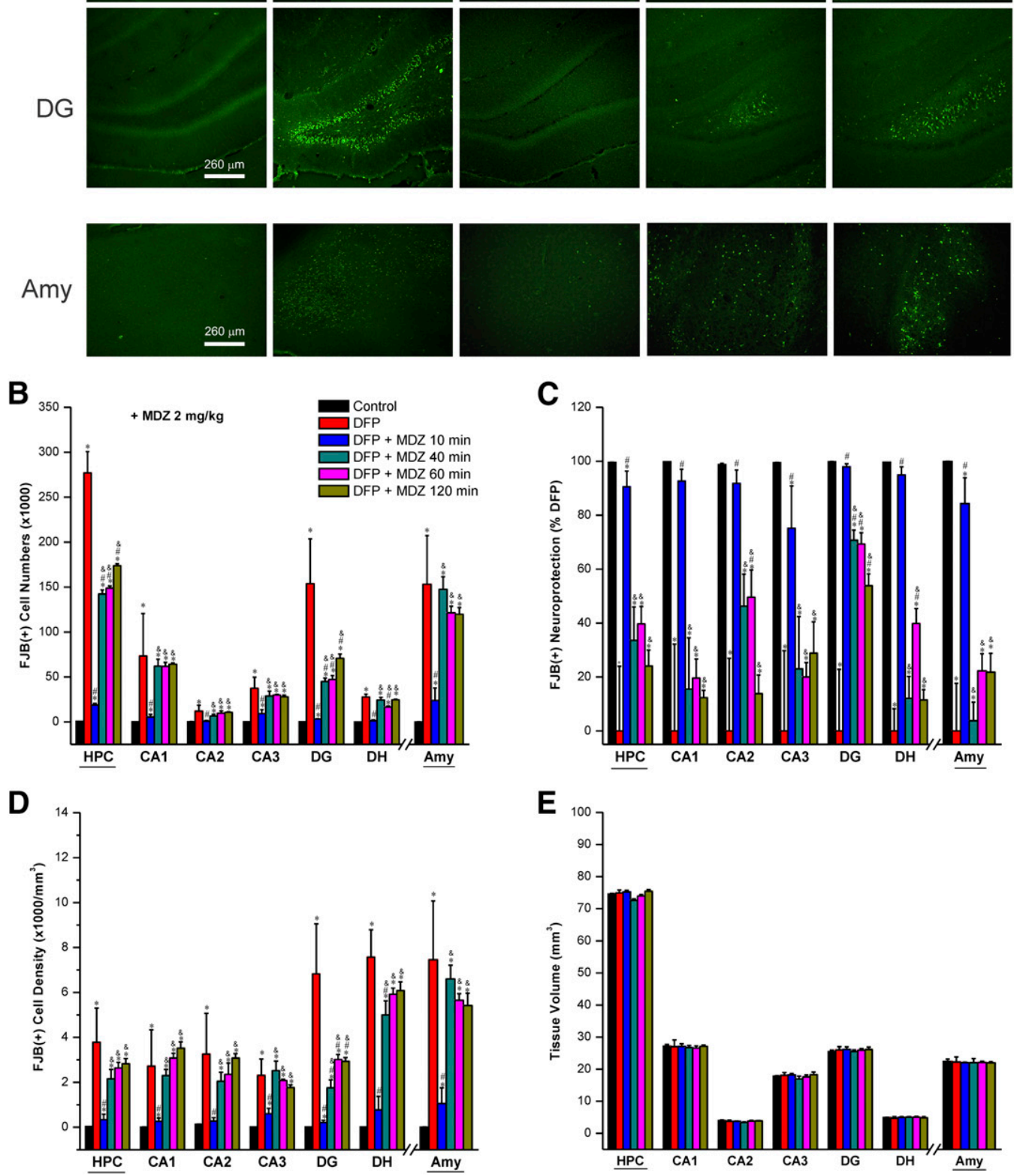

Fig. 4. Time-course protective profile of midazolam $(2 \mathrm{mg} / \mathrm{kg}$ ) on DFP-induced neuronal injury in the hippocampus (HPC) subfields and amygdala (AMY) at 3 days after DFP exposure. (A) Representative fluoro-Jade B (FJB+) staining depicting dying neurons in brain sections from animals treated with midazolam at 10-120 minutes after DFP exposure. (B) The bar charts depict FJB(+) neuronal counts by stereology in hippocampus subfields and amygdala. (C) Normalized percentage of neuroprotection by midazolam with reference to FJB(+) cells in DFP group. Normalized neuroprotection was calculated using the untreated DFP-exposed group as the baseline ( $0 \%$ protection). In this estimate, the control group not exposed to DFP was rated as $100 \%$ protected because of lack of any $\mathrm{FJB}(+)$ cells in any region. All midazolam-treated DFP-exposed rats exhibited $<100 \%$ protection. (D) FJB(+) cell density by stereology in hippocampal subfields and amygdala. (E) Brain slice thickness/volume in DFP alone and DFP + midazolam groups. Control sections (no DFP cohort) showed no FJB $(+)$ cells. Each bar represents the mean \pm S.E.M. $\left(n=4-11\right.$ per group). ${ }^{*} P<0.05$ versus control (no DFP); ${ }^{*} P<0.05$ versus DFP 
bilateral degeneration of 0.6 million $\mathrm{NeuN}(+)$ neurons ( $40 \%$ loss) was evident in the whole hippocampus (CA1, CA2, CA3, and DG subfields) after DFP exposure (Fig. 6B). The DFPinduced neurodegeneration in both hemispheres of the brain was symmetrical but highly variable within the hippocampal subfields (Fig. 6B). There was minimal or little neuronal damage in the group treated with $\mathrm{MDZ}$ at 10 minutes after exposure to DFP (Fig. 6, B and C). However, late therapy with MDZ (40, 60, or 120 minutes after DFP) provided limited or reduced neuroprotection in the hippocampus subfields as evident from the significant decrease in absolute cell numbers (Fig. 6B), and percent of surviving neurons (Fig. 6C) when compared with DFP alone and MDZ 10-minute groups. The slice thickness/volumes were similar between all DFP groups (data not shown). A similar pattern of decreased $\mathrm{NeuN}(+)$ surviving neurons was observed in extrahippocampus regions including amygdala, thalamus, piriform cortex, somatosensory cortex, and entorhinal cortex using neuropathy scoring (data not shown; see Fig. 10). Thus, these results indicate that postexposure administration of MDZ at 40 minutes or later after OP intoxication had reduced neuroprotection of principle neurons in the brain.

Effect of Midazolam on DFP-Induced Acute Degeneration of $\mathbf{P V}(+)$ Interneurons. To test whether MDZ therapy protects against interneuron loss, $\mathrm{PV}(+)$ GABAergic interneurons in the hippocampus were quantified by stereology (Fig. 7). The PV(+) interneurons were present in the granule cell layer of the dentate gyrus and strata oriens, hilus, pyramidale, and radiatum of the CA1, CA2, and CA3 subfields (Fig. 7A). The total PV(+) interneuron number in control rat hippocampus including CA1, CA2, CA3, and DG subfields was 53,362 (Fig. 7B). DFP exposure was associated with massive $(\sim 30 \%)$ loss of $\mathrm{PV}(+)$ interneurons in the hippocampus. The extent of interneuron loss was higher in the CA1 (34\%), CA3 (43\%), and DG (31\%) subfields.

Midazolam therapy at the early time point (10 minutes) was associated with significant protection against DFP-induced interneurons loss (Fig. 7, B and C). The PV(+) cell numbers (Fig. 7B), and percentage of surviving interneurons (Fig. 7C) were significantly lower in the MDZ subgroups $(40,60$, and 120 minutes) compared with to the DFP alone and 10-minute MDZ groups (Fig. 7C), indicating lack of protection by late MDZ therapy. There was no change in the slice thickness/volumes (data not shown). A significantly reduced $\mathrm{PV}(+)$ surviving neuronal distribution was noted in extrahippocampus regions including amygdala, thalamus, piriform cortex, somatosensory cortex, and entorhinal cortex (see Fig. 10).

Thus, these results indicate that delayed therapy (40 minutes or later) with MDZ after OP intoxication offers limited protection in preserving the $\mathrm{PV}(+)$ interneurons in the brain.

Effect of Midazolam on DFP-Induced Acute GFAP(+) Astrogliosis and Neuroinflammation. Exposure to OP agents such as sarin or DFP produces massive activation of the neuroinflammatory response (Zimmer et al., 1997; Li et al., 2015; Sisó et al., 2017; Liang et al., 2018). It is associated with astrocytosis and microglial activation in hippocampus, amygdala, and many other brain regions. To examine whether MDZ therapy protects against excessive astrocytic response,
GFAP(+) immunostaining was quantified in the hippocampus and amygdala. Astrocytic reactions were significantly increased (166\%-205\%) at 3 days after DFP exposure in the hippocampus and amygdala (Fig. 8, A and B). A typical astrocytic reaction includes swollen soma and shorter processes in the hippocampus and amygdala regions (Fig. 8B). Early therapy with MDZ (10 minutes after DFP exposure) was associated with significant reduction of GFAP $(+)$ expression in both the hippocampus and amygdala regions (Fig. 8C). However, there was little change in $\mathrm{GFAP}(+)$ expression in rats treated with $\mathrm{MDZ}$ at 40 minutes or later after exposure to DFP in brain regions examined, with an exception of the DG in MDZ 40-minute subgroup that exhibited a significant reduction in reactive $\mathrm{GFAP}(+)$ astrocytes (Fig. 8, C and D). These results indicate that late therapy (40 minutes or later) with MDZ was not associated with limited reduction in astrocyte inflammatory response after OP intoxication.

Effect of Midazolam on DFP-Induced Acute IBA1(+) Microgliosis and Neuroinflammation. To examine whether MDZ protects against DFP-induced microglial activation at early and late therapy, IBA1(+) immunostaining was analyzed in the hippocampus and amygdala (Fig. 9, A and B). The activated microglial response appeared as enlarged soma as well as shorter and stouter processes than normal microglial cells (Fig. 9B). There was a significant microglial activation (140\%-208\%) in the hippocampal CA1, CA3, and DG subfields and amygdala at 3 days after exposure to DFP (Fig. 9, C and D). Early therapy with MDZ (10 minutes after DFP exposure) was associated with significant reduction in IBA1(+) expression in the hippocampus subfields but not in the amygdala (Fig. 9, C and D). In the rats treated with MDZ at delayed time points $(40,60$, or 120 minutes after DFP exposure), there was limited protection against DFPinduced IBA1 $(+)$ microgliosis (Fig. 9, C and D), except a significant reduction in DG in MDZ 40-minute subgroup versus DFP-alone group (Fig. 9C). Thus, these results indicate that late therapy with MDZ was associated with minimal protection against DFP-induced microglial inflammatory response in the brain.

Spatiotemporal Correlation and Mixed-Effects Analysis of Early and Late Therapy with Midazolam on DFP-Induced Neuronal Injury, Neurodegeneration, and Neuroinflammation. To examine whether time latency for MDZ administration after DFP exposure is correlated with neuropathology severity (\% of DFP alone) across brain regions from the same set of animals, we performed linear repeated mixed-effects regression models to account for within-animal correlation (Fig. 10). Three metrics of neuropathology associated with DFP intoxication were analyzed using spatiotemporal linear correlation and mixed-effects regression: 1) $\mathrm{FJB}(+)$ neuronal injury, 2) $\mathrm{NeuN}(+)$ and $\mathrm{PV}$ $(+)$ neurodegeneration, and 3) GFAP $(+)$ astrogliosis and IBA1 $(+)$ microgliosis as cellular neuroinflammation.

Unlike generalized linear models where observations are assumed independent with fixed effects without error corrections, in mixed-effects models, observations are assumed not independent and random effects are fitted to model additional source of variation with error corrections (Stroup, 2012). The mixed-effects models used for such correlation included region,

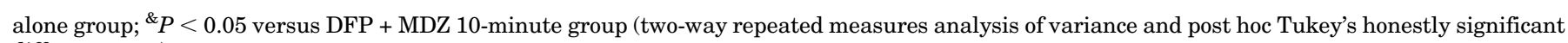
difference test). 
A

\section{Control}
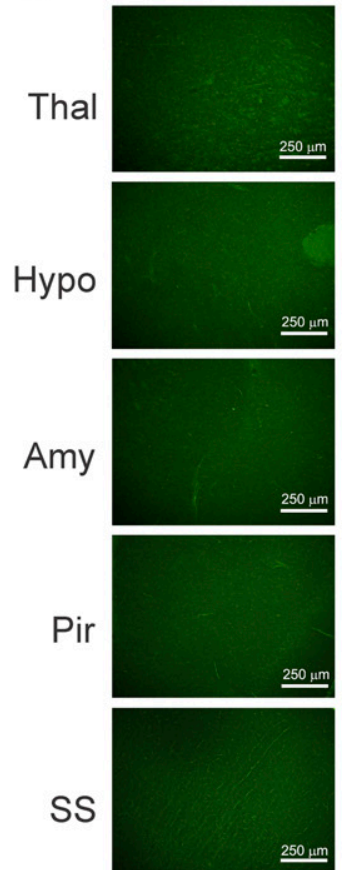

Ent

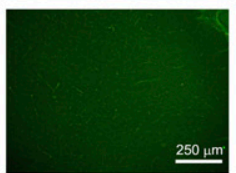

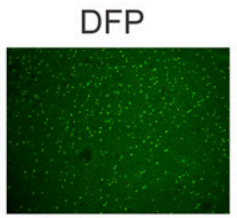
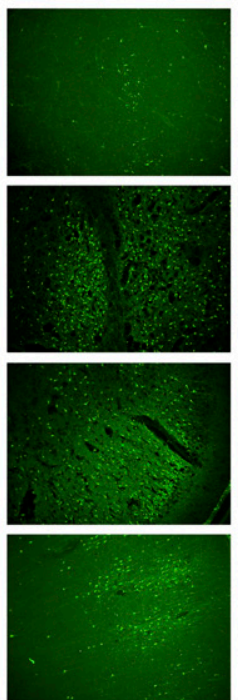

DFP+MDZ10
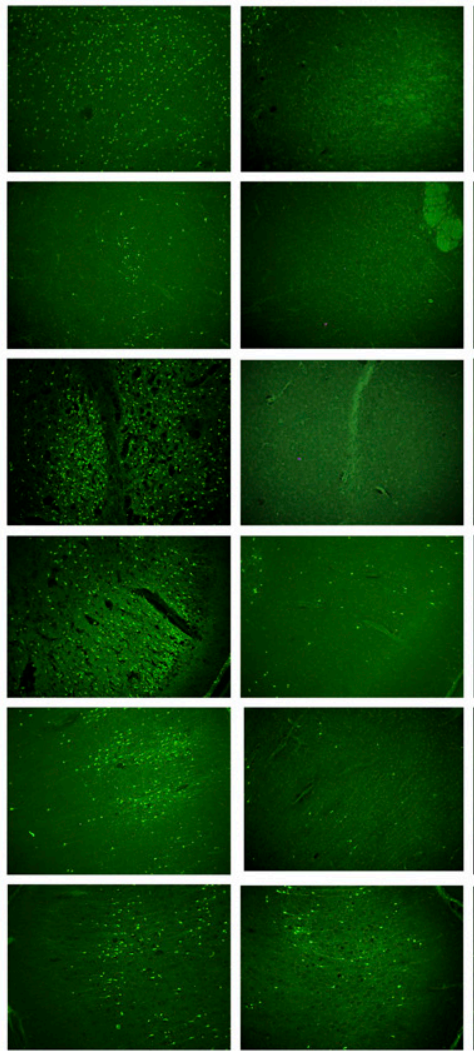

\section{DFP+MDZ40}
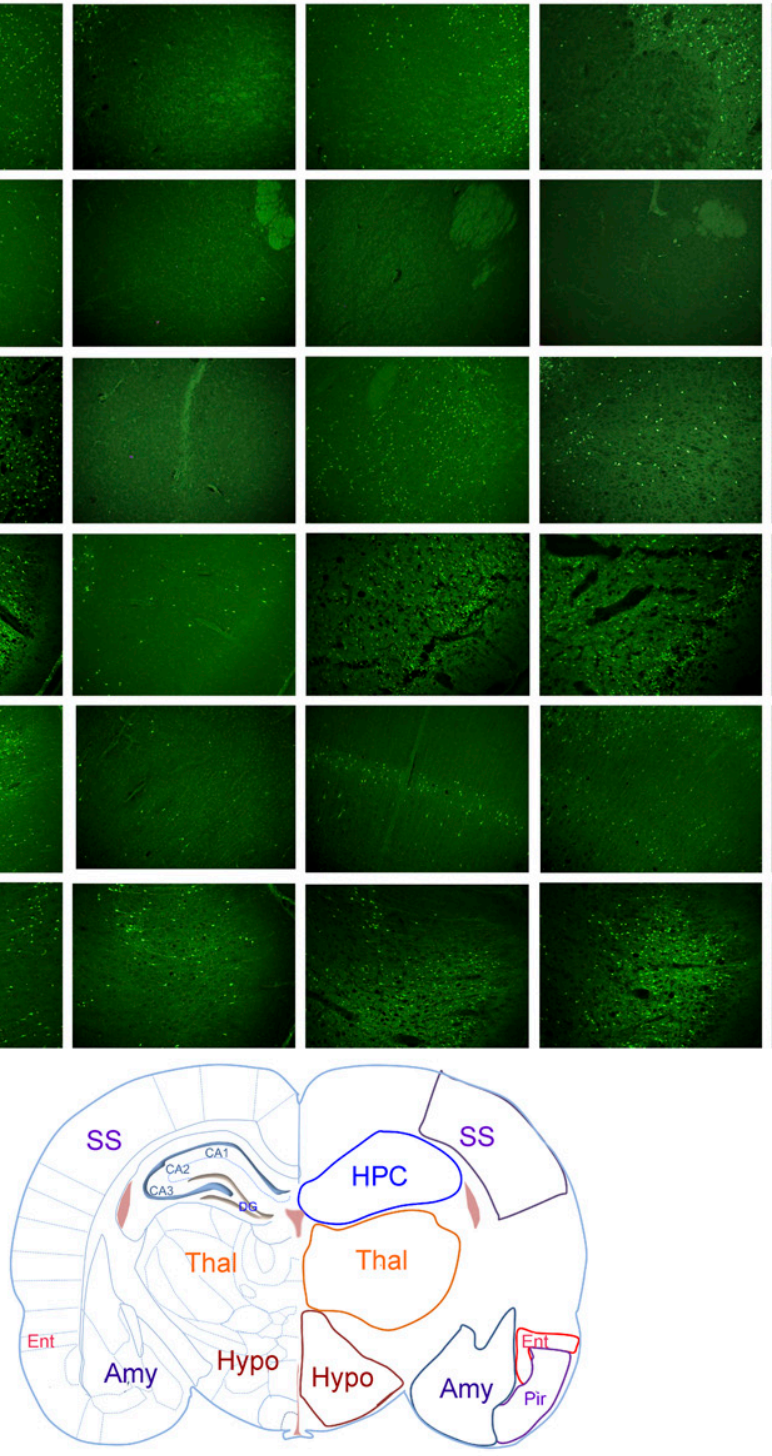

C

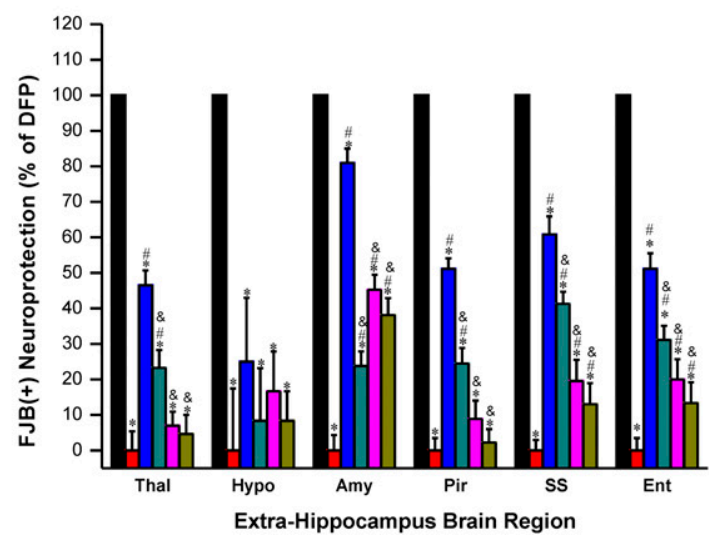

Fig. 5. Time-course profile of midazolam ( $2 \mathrm{mg} / \mathrm{kg}$, i.m.) on DFP-induced neuronal injury in various extrahippocampal regions at 3 days after DFP exposure. (A) Representative FJB(+) staining depicting dying neurons in extrahippocampal regions in control and DFP subgroups. Rat brain atlas illustrating the regions selected for analysis of $\mathrm{FJB}(+)$ staining, including thalamus (Thal), hypothalamus (Hypo), amygdala (Amy), piriform cortex (Pir), somatosensory cortex (SS), and entorhinal cortex (Ent) regions. (B) The bar charts depict neuropathology scores in these regions and represent severity level of FJB(+) staining dying neurons. DFP exposures was associated with severe damage with high neuropathology score. (C) Normalized percent neuroprotection in extrahippocampal brain regions from rats treated with midazolam at 10-120 minutes after DFP exposure. Normalized neuroprotection was calculated using the untreated DFP-exposed group as the baseline (0\% protection). Each bar represents the mean \pm S.E.M. $\left(n=4-11\right.$ rats per group). ${ }^{*} P<0.05$ versus control (no DFP); $P<0.05$ versus DFP group; ${ }^{\circledR} P<0.05$ versus DFP+MDZ 10 -minute group (Mann-Whitney $U$ test). 

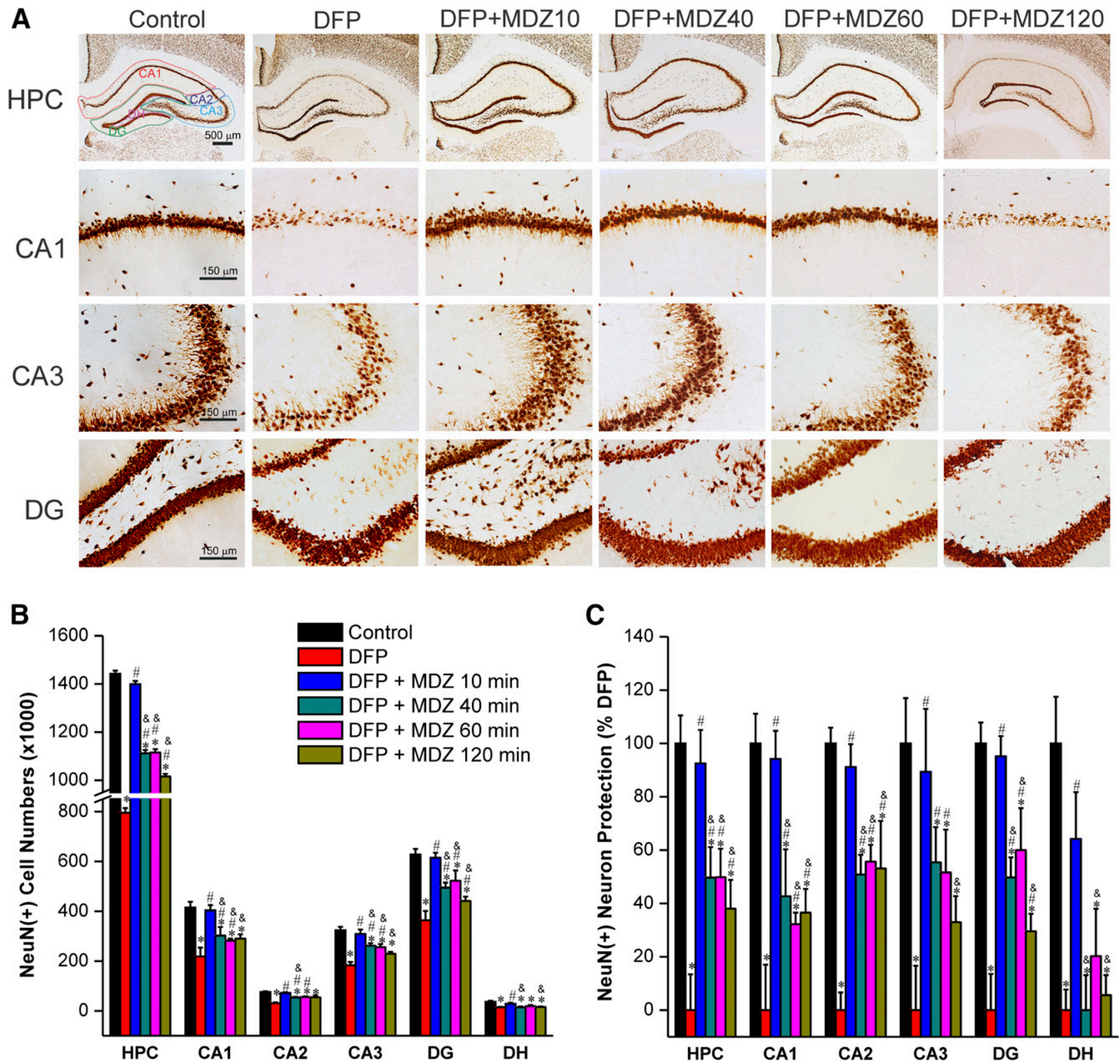

Fig. 6. Time-course profile of midazolam ( $2 \mathrm{mg} / \mathrm{kg}$, i.m.) on DFP-induced loss of NeuN(+) principle neurons in the hippocampus (HPC) and its subfields at 3 days after DFP exposure. (A) Representative sections of $\mathrm{NeuN}(+)$ immunostaining in brain section from animals treated with midazolam at 10 , 40 , 60 , or 120 minutes after DFP exposure. (B) The bar charts depict stereologic quantification of absolute NeuN(+) cell numbers in the hippocampus subfields. (C) Percentage of protection of $\mathrm{NeuN}(+)$ cell loss in the hippocampal subfields in midazolam groups. Normalized neuroprotection of NeuN(+) surviving neurons is calculated using untreated DFP-exposed group as $0 \%$ protection. In this estimate, the control group not exposed to DFP was rated as $100 \%$ surviving cells because of lack of any $\mathrm{NeuN}(+)$ neuron loss in this group in any region. All midazolam-treated DFP-exposed rats exhibited $<100 \% \mathrm{NeuN}(+)$ surviving cells. Values represent the mean \pm S.E.M. $\left(n=4-11\right.$ rats per group). ${ }^{*} P<0.05$ versus control (no DFP); ${ }^{\#} P<0.05$ versus DFP group; ${ }^{\&} P<0.05$ versus DFP+MDZ 10-minute group (two-way repeated measures analysis of variance and post hoc Tukey's honestly significant difference test).

latency time for MDZ administration, and the interactions between regions and latency time. Using these models, the latency time of MDZ's median peak neuroprotection was calculated by deriving the estimated latency time for MDZ treatment at which the maximum neuroprotection occurred both overall and within each brain region.

Scatterplots were generated illustrating the relationship between average regional protection or sum of regional (global) protection and the time for MDZ treatment. Boxed plots were generated with an estimated peak effect and $95 \%$ confidence interval for the latency time of peak neuronal damage severity.
A repeated-measures analysis of the temporal progression of $\mathrm{FJB}(+)$ neuronal injury (Fig. 10, A-C) suggested that moderate to severe damage was observed with early (10 minutes) and late (40 minutes or later) time points in many brain regions (Fig. 10A). Peak protection was observed in 10-minute group with strong regional variation in the extent of neuroprotection (Fig. 10A), especially in the amygdala, somatosensory cortex, and hippocampus. A regression analysis showed a strong association $\left(R^{2}=0.96\right)$ between the time for MDZ therapy and global neuronal damage (Fig. 10B). The estimated peak protection of $\mathrm{FJB}(+)$ damage was observed in the 
A
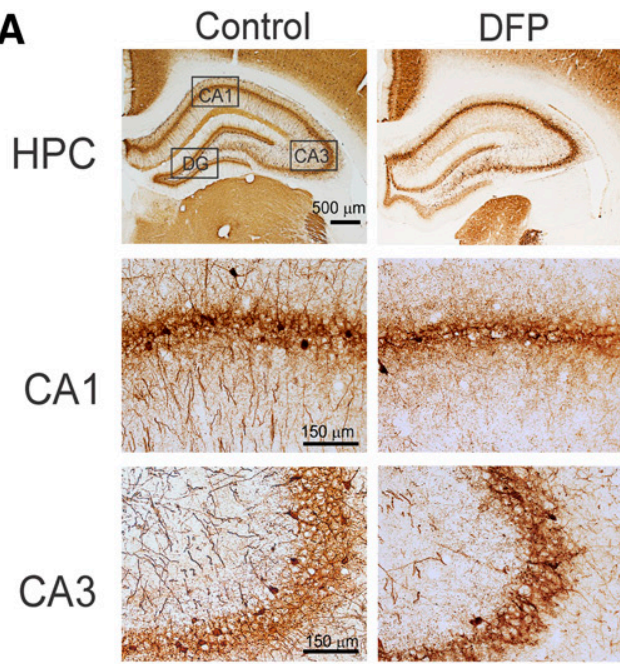

DG
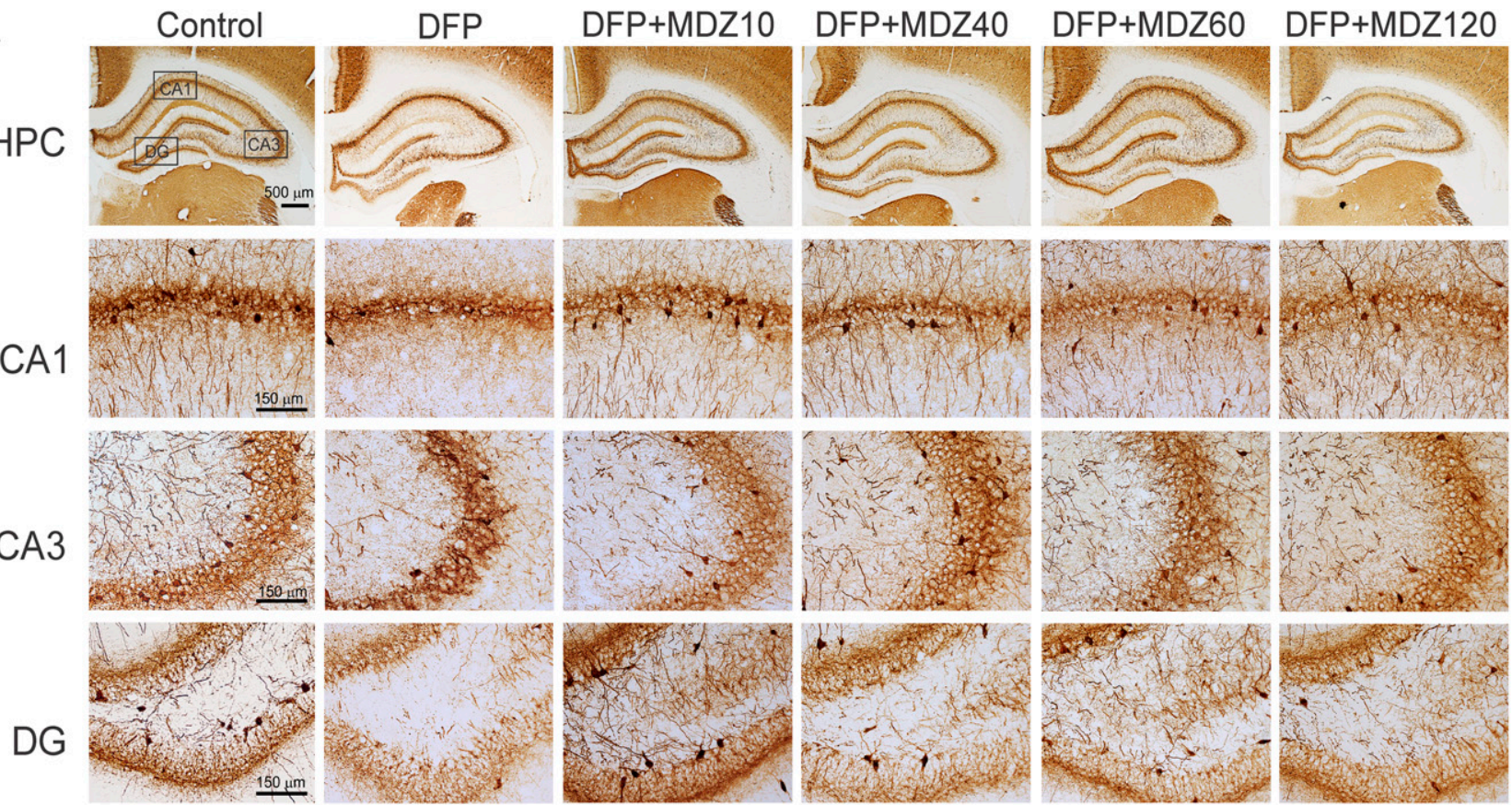

B

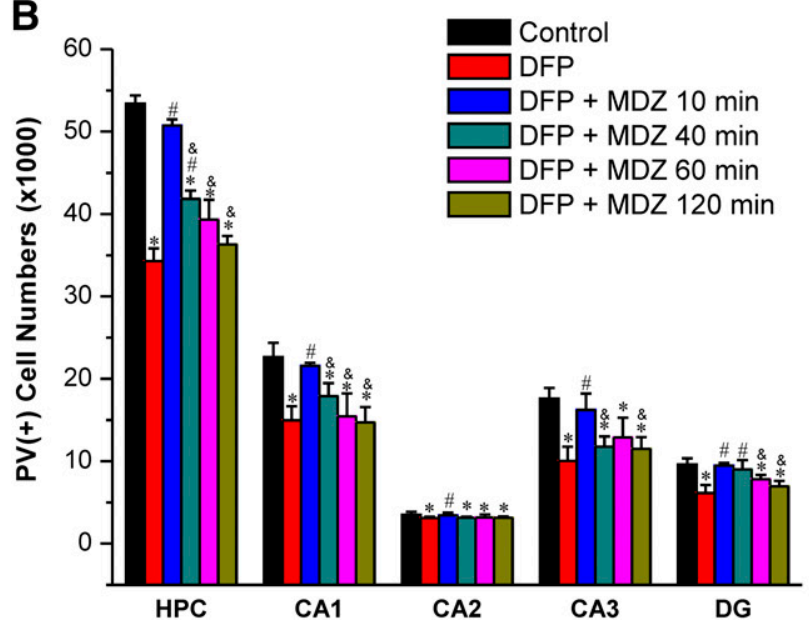

C

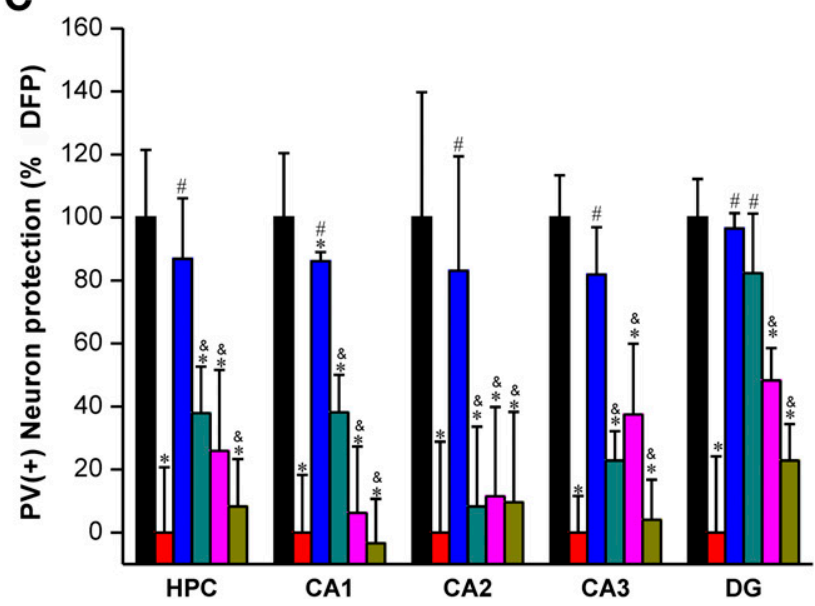

Fig. 7. Time-course profile of midazolam ( $2 \mathrm{mg} / \mathrm{kg}$, i.m.) on DFP-induced loss of PV(+) interneurons in the hippocampus (HPC) and its subfields at 3 days after DFP exposure. (A) Representative sections of PV(+) immunostaining in the brain sections from midazolam-treated groups. (B) The bar charts depict stereologic quantification of absolute $\mathrm{PV}(+)$ cell numbers in the hippocampus subfields in the control and midazolam groups. (C) Percentage of protection of $\mathrm{PV}(+)$ cell loss in various subgroups in the hippocampal subfields. Normalized neuroprotection of $\mathrm{PV}(+)$ surviving interneurons is calculated using untreated DFP-exposed group as $0 \%$ protection. In this estimate, the control group not exposed to DFP was rated as $100 \%$ surviving cells because of lack of any $\mathrm{PV}(+)$ interneuron loss in this group in any region. All midazolam-treated DFP-exposed rats exhibited $<100 \%$ PV $(+)$ surviving interneurons. Values represent the mean \pm S.E.M. $\left(n=4-11\right.$ rats per group). ${ }^{*} P<0.05$ versus control (no DFP); $P<0.05$ versus DFP group; ${ }^{\&} P<0.05$ versus DFP+MDZ 10-minute group (two-way repeated measures analysis of variance and post hoc Tukey's honestly significant difference test).

10-minute group. A progressive and significant decrease in protection $(P<0.05)$ was observed with further delay in MDZ treatment (Fig. 10C).

For correlation analysis of neurodegeneration (Fig. 10, D-F), an average neurodegeneration protection was derived from $\mathrm{NeuN}(+)$ and $\mathrm{PV}(+)$ cell loss. Analysis of the percentage of protection data suggested that time of MDZ administration influenced the extent of neurodegeneration (Fig. 10D). Moreover, animals that received MDZ at delayed time points had a significantly lower number of surviving neurons than those with an early (10 minutes) time point (Fig. 10E). The neurodegeneration severity corresponded globally to the delayed time of drug administration (Fig. 10F).
In addition, analysis of the temporal progression of cellular neuroinflammation (Fig. 10, G-I), as derived from the average $\mathrm{GFAP}(+)$ astrogliosis and IBA1(+) microgliosis, suggested moderate to strong protection that varies with the time for therapy and also across brain regions (Fig. 10G). There was a strong linear relationship $\left(R^{2}=0.96\right)$ between the time for drug therapy and global neuroinflammation severity (Fig. 10H). A progressive decline in neuroinflammation protection was evident in proportion to the delayed time for MDZ administration (Fig. 10I).

The above three neuropathology metrics (neuronal injury, neurodegeneration, and neuroinflammation) were evaluated across regions using linear, mixed-effects regression analysis 
A

A

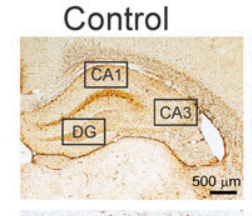
DFP

HPC

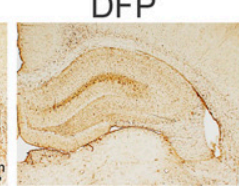

CA1
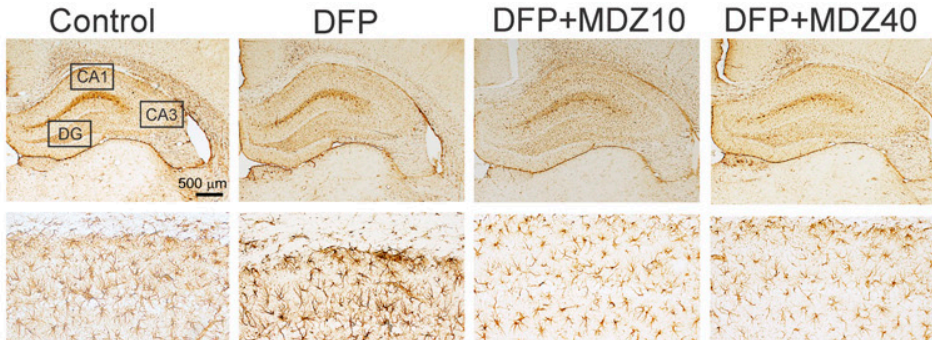

DFP+MDZ60

DFP+MDZ120

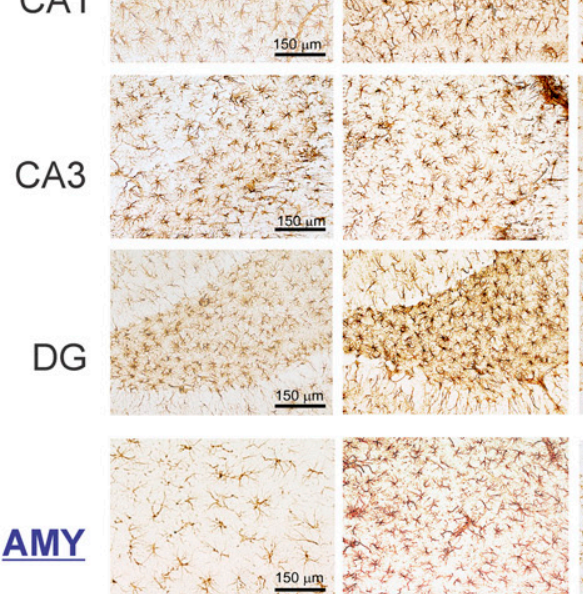

B

Control
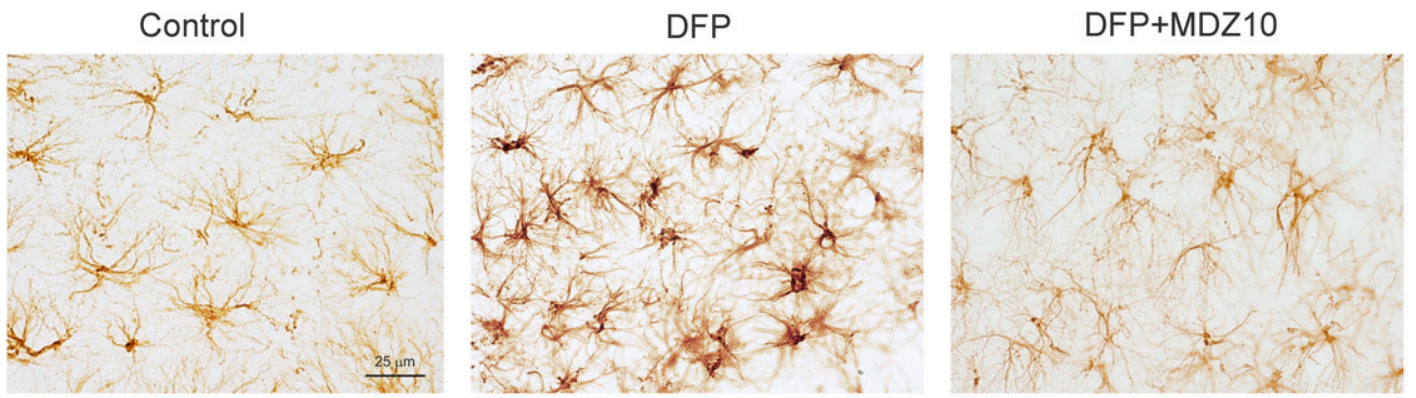

C

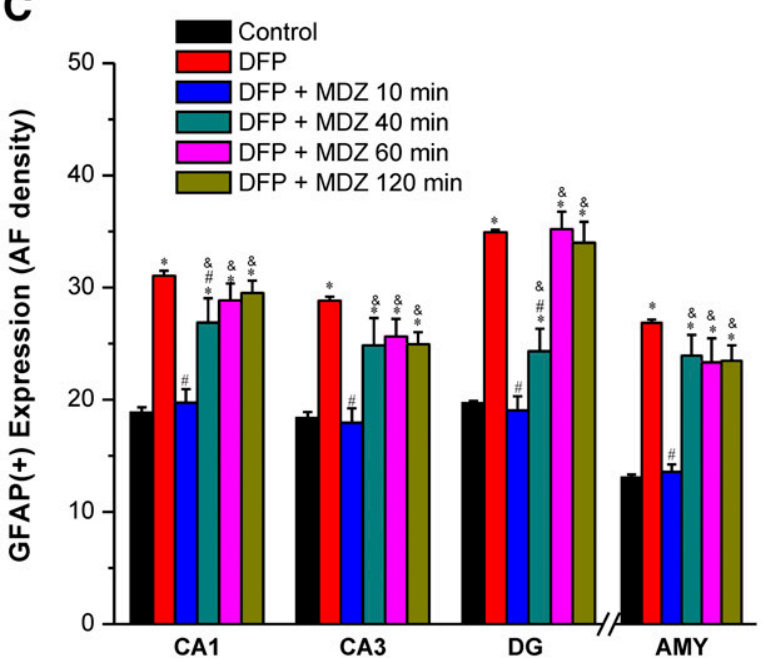

D

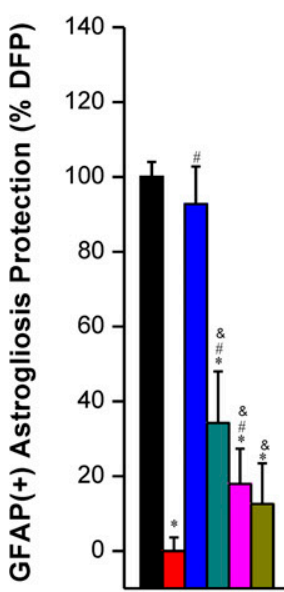

CA1

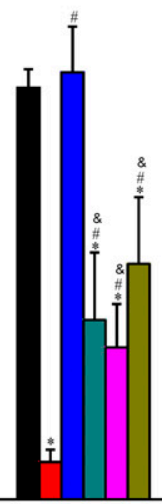

CA3

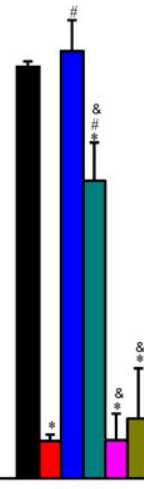

DG

Fig. 8. Time-course of protective effect of midazolam $(2 \mathrm{mg} / \mathrm{kg})$ on DFP-induced GFAP $(+)$ astrocyte neuroinflammation in the hippocampus $(\mathrm{HPC})$ and amygdala (AMY) regions at 3 days after DFP exposure. (A) Representative sections of GFAP $(+)$ immunostaining in the hippocampus and amygdala. (B) A typical reactive astrocyte image includes swollen soma and shorter processes with increased AF density in the hippocampal regions in the untreated DFP group. (C) The bar charts depict the area fractionation (AF) densitometric quantification of GFAP $(+)$ expression in the hippocampal subfields and amygdala. (D) Percentage of protection of GFAP $(+)$ astrogliosis in various subgroups in the hippocampus subfields. Normalized protection of GFAP $(+)$ neuroinflammation is calculated using untreated DFP-exposed group as $0 \%$ protection. In this estimate, the control group not exposed to DFP was rated as $100 \% \mathrm{GFAP}(+)$ expressing astrocytes because of lack of any damaging response in this group in any region. Values represent the mean \pm S.E.M. $\left(n=4-11\right.$ rats per group). ${ }^{*} P<0.05$ versus control (no DFP); ${ }^{\#} P<0.05$ versus DFP group; ${ }^{*} P<0.05$ versus DFP+MDZ 10 -minute group (two-way repeated measures analysis of variance and post hoc Tukey's honestly significant difference test). 
A Control

\section{DFP}

HPC
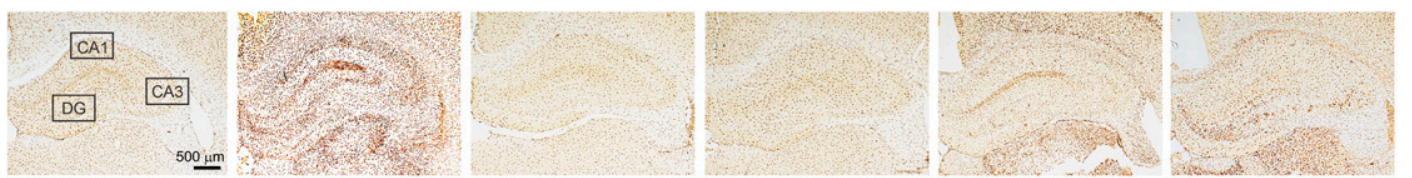

CA1

CA3

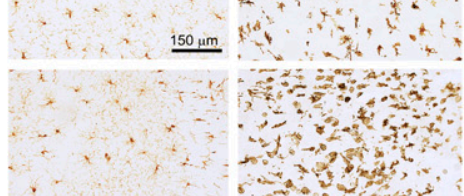

DG

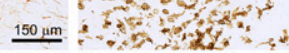

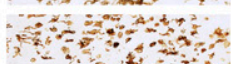

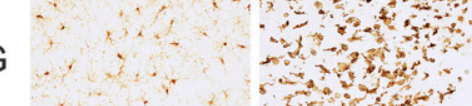

$150 \mathrm{~m}, 5 \mathrm{n}, \mathrm{s}$

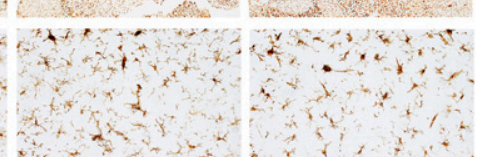

$\underline{A M Y}$

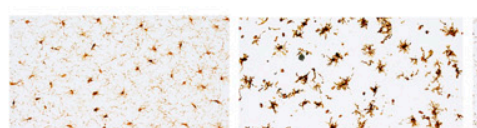
150 um

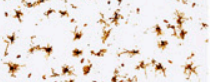

B

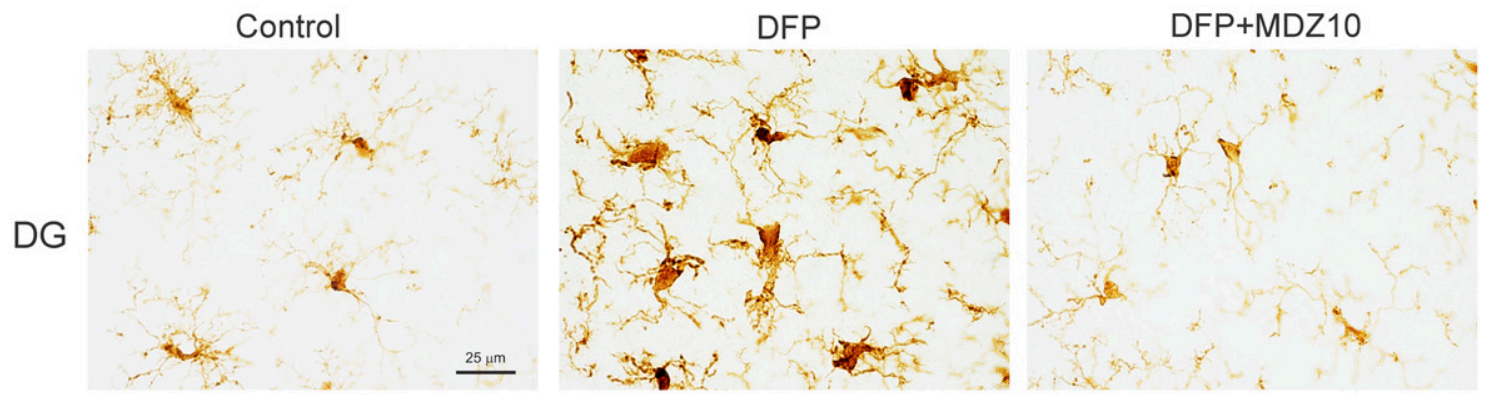

C
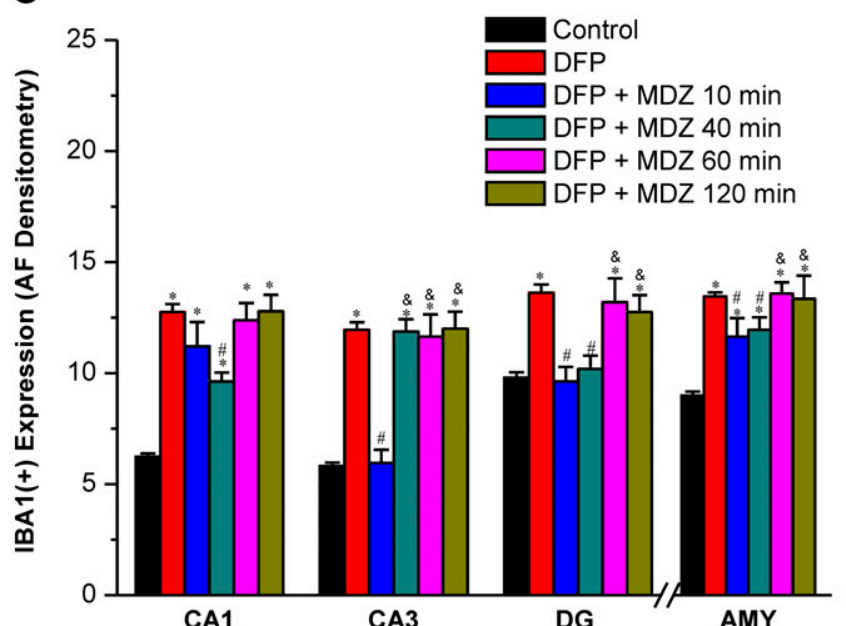

D

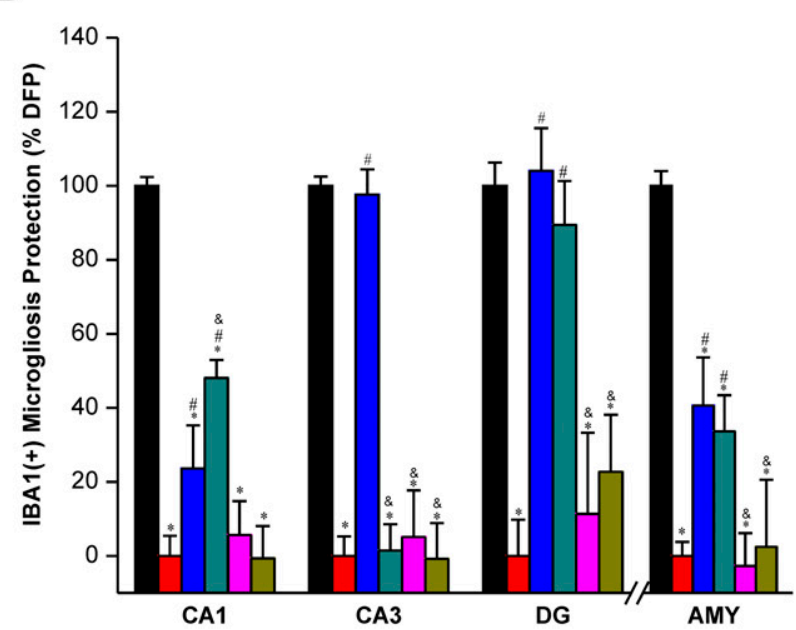

Fig. 9. Time-course of protective effect of midazolam $(2 \mathrm{mg} / \mathrm{kg})$ on DFP-induced IBA1(+) microglial neuroinflammation in the hippocampus (HPC) and amygdala (AMY) regions at 3 days after DFP exposure. (A) Representative sections of IBA1(+) immunostaining in the hippocampus and amygdala in control and midazolam groups. (B) The activated microglial responses appear as enlarged soma as well as shorter and stouter processes than normal microglial cells in the untreated DFP group. (C) The bar graphs depict area fractionation (AF) densitometric quantification values of IBA1(+) expression in the hippocampus subfields and amygdala. (D) Percentage of protection of IBA1(+) microgliosis in various subgroups in the hippocampus subfields and amygdala. Normalized protection of IBA1(+) neuroinflammation is calculated using untreated DFP-exposed group as $0 \%$ protection. In this estimate, the control group not exposed to DFP was rated as 100\% IBA1(+) expressing microglia because of lack of any glial activation response in this group in any region. Values represent the mean \pm S.E.M. ( $n=4-11$ rats per group). $* P<0.05$ versus control (no DFP); ${ }^{*} P<0.05$ versus DFP group; ${ }^{\&} P<0.05$ versus DFP+MDZ 10-minute group (two-way repeated measures analysis of variance and post hoc Tukey's honestly significant difference test). 
A

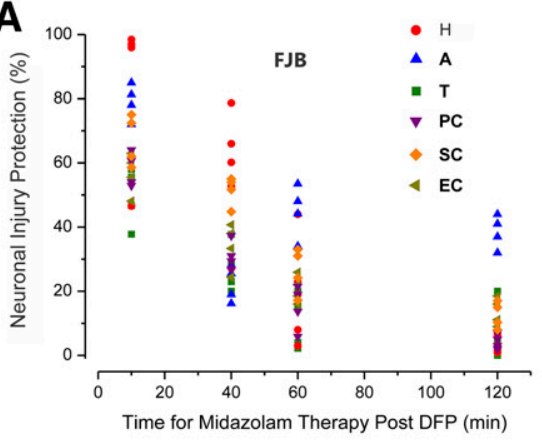

D

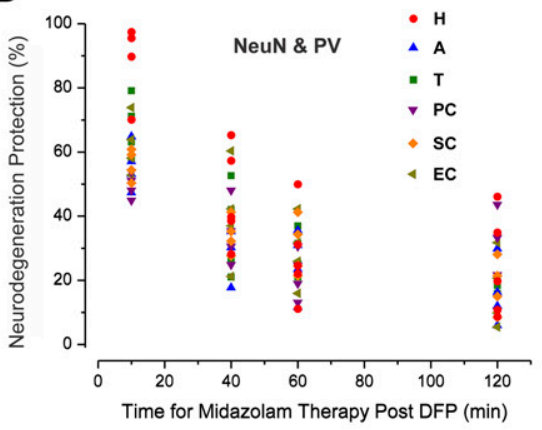

G
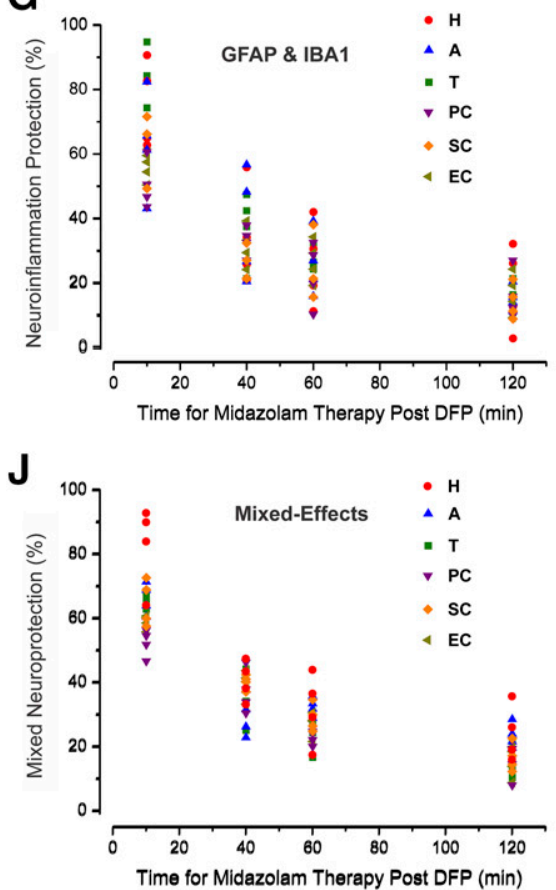

B

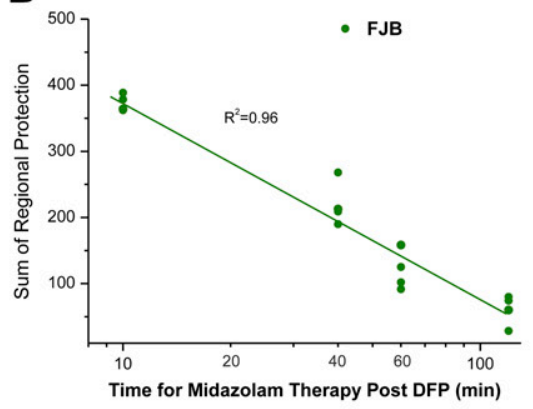

E

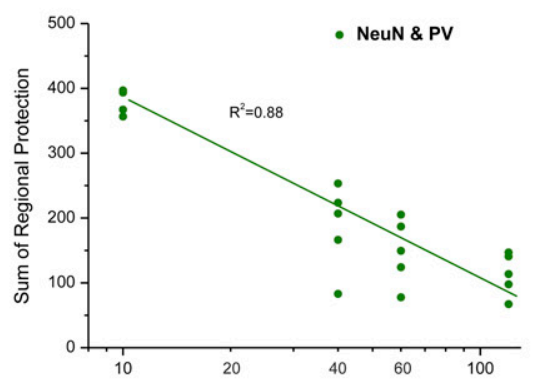

Time for Midazolam Therapy Post DFP ( $\mathrm{min})$

H

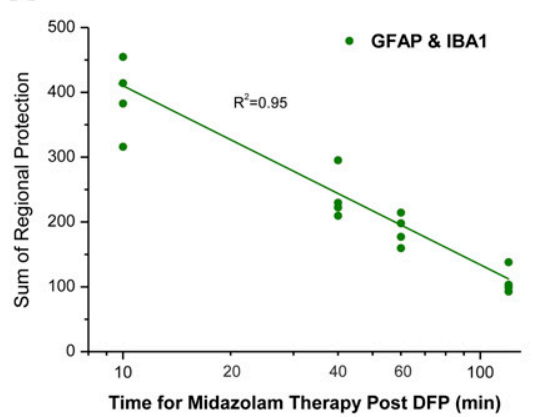

K

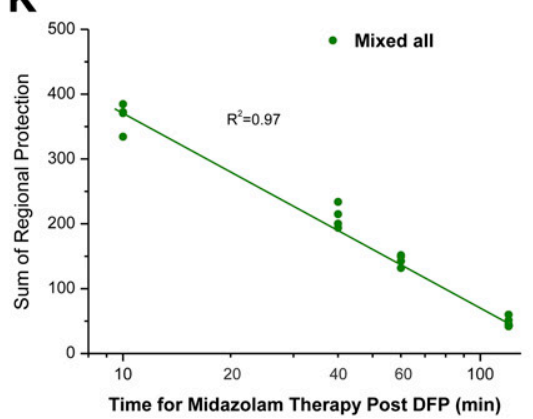

C

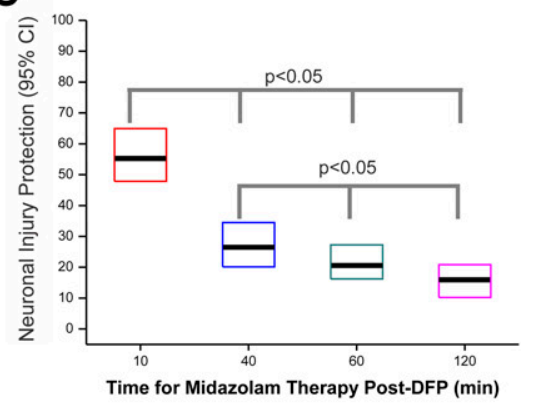

F

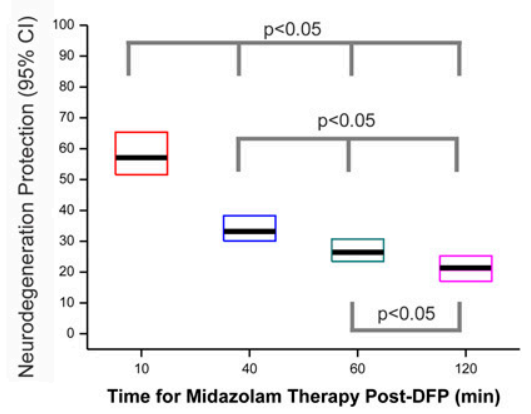

I

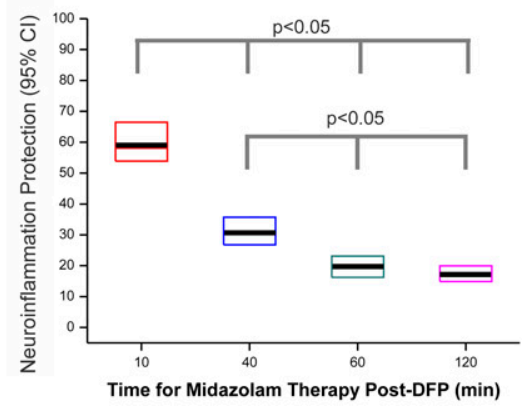

L

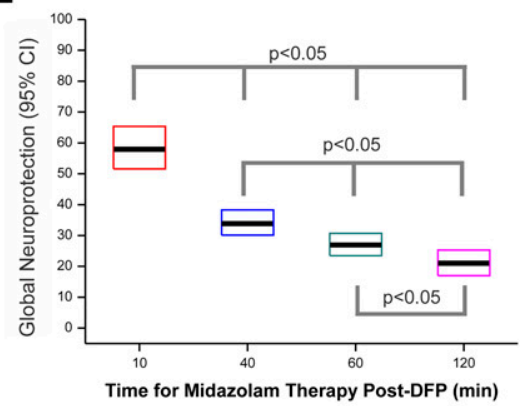

Fig. 10. Temporal correlation of neuropathology after midazolam treatment $(2 \mathrm{mg} / \mathrm{kg}$ ) in rats exposed to DFP. (A-C) Spatiotemporal correlation of the extent of protection against $\mathrm{FJB}(+)$ neuronal injury in midazolam-treated cohorts after acute DFP exposure. (A) Scatterplot of average percentage of neuroprotection in various brain regions. Each individual symbol represents data from a single animal. Each plot representing a different brain region was fitted on data from the 17 animals that received midazolam at the time points of 10 minutes $(n=4), 40$ minutes $(n=5), 60$ minutes $(n=4)$, and 120 minutes $(n=4)$. (B) Scatterplot of global neuroprotection, as modeled using sum of regional protection. Each individual symbol represents data from a single animal. The curve was fitted on data from the 17 animals that received midazolam at various time points. (C) Box plots indicating estimated global median peak protection (horizontal black bar) and 95\% confidence intervals (CI) of data in (B). (D-F) Spatiotemporal correlation of the extent of protection against $\mathrm{NeuN}(+)$ and $\mathrm{PV}(+)$ cell loss in midazolam-treated cohorts after acute DFP exposure. (D) Scatterplot of average neurodegeneration protection in various brain regions. Each individual symbol represents data from a single animal. Each plot representing a different brain region was fitted on data from the 17 animals that received midazolam at various time points. (E) Scatterplot of global neurodegeneration protection, as modeled using sum of regional protection. Each individual symbol represents data from a single animal. The curve was fitted on data from the 17 animals that received midazolam at various time points. (F) Box plots indicating estimated global peak neurodegeneration protection and 95\% CI of data in (E). (G-I) Spatiotemporal correlation of the extent of protection against GFAP $(+)$ and IBA1(+) neuroinflammation in midazolam-treated cohorts after acute DFP exposure. (G) Scatterplot of average neuroinflammation protection in various brain regions. Each individual symbol represents data from a single animal. $(\mathrm{H})$ Scatterplot of global neuroinflammation protection, as modeled using sum of regional protection. Each individual symbol represents data from a single animal. (I) Box plots indicating estimated global peak neuroinflammation protection and 95\% CI of data in (H). (J-L) Spatiotemporal 
(Fig. 10, J-L). There were striking differences in the mixed neuroprotection in various brain regions across MDZ treatment times. There was a strong correlation between latency for MDZ treatment and global neuroprotection $\left(R^{2}=0.97\right.$, $P<0.001$ ), indicating significantly diminished neuroprotection with late therapy at 40 minutes or later after DFP exposure (Fig. 10K). Groups with delayed MDZ administration (40 minutes or later) exhibited progressively significant reduction in global neuroprotection $(P<0.05)$ compared with the early therapy (10 minutes) group (Fig. 10L).

Overall, delayed MDZ administration (40,60, or 120 minutes) was significantly $(P<0.05)$ associated with more severe brain damage, which could lead to the long-term neurologic sequelae of acute $\mathrm{OP}$ intoxication.

\section{Discussion}

We used the DFP exposure model in rats to characterize SE and brain damage after early and late MDZ therapy. It has long been known that MDZ protects against seizures and neuropathology in nerve agent models (McDonough and Shih, 1997; McDonough et al., 1999). Using a common model of $\mathrm{OP}$ intoxication, with a realistic therapeutic window for drug administration in the scenario of a chemical attack (40 minutes or later), this study found a marked reduction in anticonvulsant efficacy of MDZ. In addition, there was a progressive decrease in protection against DFP-induced neuronal injury in the cortex, hippocampus, and amygdala. Furthermore, the pattern of neurodegeneration caused by DFP was accompanied by an astrocytic response and the activation of microglia. Taken together these observations confirm the refractoriness to benzodiazepines, whereby the effectiveness of MDZ may be less at later administration times, resulting in seizure recurrence, neuroinflammation, and severe brain damage.

We used DFP as a surrogate chemical agent because it replicates many features of nerve agent neurotoxicity due to its chemical and mechanistic similarities (Deshpande et al., 2010; Jett and Yeung, 2010; Pouliot et al., 2016; Sisó et al., 2017). DFP (diisopropylfluorophosphate) is structurally very similar to the phosphonofluoridate-type (G-class) nerve agents GB (sarin; $(R S)$ propan-2-yl methylphosphonofluoridate) and GD (soman; 3,3dimethylbutan-2-yl methylphosphonofluoridate). The average latency of 8-10 minutes for onset of seizures after DFP exposure in rats is consistent with results from nerve agent models such as soman and sarin (Shih and McDonough, 1999; Apland et al., 2014). The brain damage after SE is a time-dependent process with greater injury occurring over an elapsed time of seizure activity (McDonough et al., 1995; Kim et al., 1999; Li et al., 2011). In the present study, MDZ rapidly terminated EEG and behavioral seizures when administered early. However, MDZ-treated animals exhibited seizure recurrence after initial suppression, indicating the time-dependent emergence of pharmacoresistant SE. These observations reaffirm a previously described limitation of benzodiazepines as anticonvulsant antidotes for OP intoxication (McDonough et al., 2010; Apland et al., 2014; Kuruba et al., 2018).

DFP induced a marked neuronal injury and neurodegeneration of principal cells and inhibitory interneurons in the hippocampus, amygdala, and cortical regions. This neuropathology data suggest that extensive loss of principal neurons and interneurons is a hallmark feature of OP neurotoxicity (Apland et al., 2010; Chen, 2012; Chen et al., 2014; Ferchmin et al., 2014; Li et al., 2015; Rojas et al., 2015; Sisó et al., 2017). Inhibitory interneurons are critical in regulating neuronal network oscillations, synchronizing principal neuron networks, and providing fidelity for synaptic transmission. $\mathrm{PV}(+)$ interneurons, which constitute about $40 \%$ of total GABAergic inhibitory interneurons, play a critical role in multiple neuronal circuits in the hippocampus and cortex.

A significant reduction in inhibitory transmission and synaptic plasticity has been reported in the hippocampus and amygdala after soman exposure (Aroniadou-Anderjaska et al., 2009; Prager et al., 2014). Early administration of MDZ is required to rescue extensive damage of principal cells and interneurons. The OP neurotoxicity, especially when administration of MDZ is delayed, is associated with massive loss of principal and interneurons which could lead to long-term neurologic risk for the development of chronic epilepsy after refractory SE and cognitive dysfunction. Moreover, there is strong correlation between the extent of seizure activity (i.e., latency time for MDZ treatment after DFP exposure) and neurodegeneration and cellular neuroinflammation (Fig. 10). These outcomes are consistent with the hallmark hippocampal sclerosis in patients with epilepsy and animal models of chronic epilepsy (Gruber et al., 1994; André et al., 2001; Kobayashi and Buckmaster, 2003; de Araujo Furtado et al., 2010; Fujikawa, 1996; Lemos and Cavalheiro, 1995).

DFP induced neuroinflammation involving activation of astrocytes and microglia response. The roles of astrocytes and microglia in brain damage are complex, including both beneficial and detrimental effects depending on spatiotemporal pattern of cytokines release (Zimmer et al., 1997; Li et al., 2015; McElroy et al., 2017; Sisó et al., 2017; Liang et al., 2018). Because microglia are the main immune cells in the brain, they have the potential to contribute to synaptic dysfunction in acute neurotoxic insults like OP intoxication. Microglia, which account for $10 \%-15 \%$ of all cells within the brain, are highly reactive to injury or neurotoxic exposure. Activation of microglia is believed to contribute to seizure activity after neuronal injury (Baille-Le Crom et al., 1995; Collombet et al., 2005; de Araujo Furtado et al., 2010; Chen, 2012). Activated microglia release deleterious cytokines such as tumor necrosis factor- $\alpha$ and interleukin, and they play a crucial role in neuronal repair and homeostasis. Persistent activation of microglia likely contributes to aberrant growth of basal dendrites in the hippocampus and creation of a recurrent excitatory circuit for seizures. The hypertrophied cells are an indication of DFP neurotoxicity leading to gliosis and disruption of the BBB

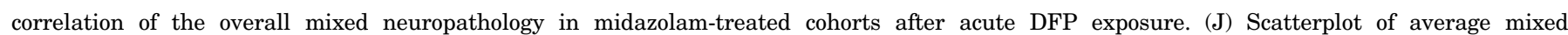

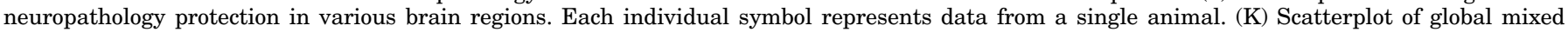

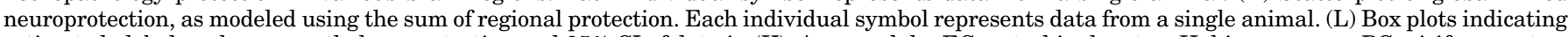

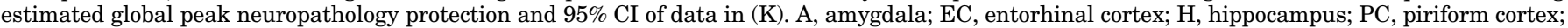
SC, somatosensory cortex; $\mathrm{T}$, thalamus. 
in the hippocampus and other regions (Petito et al., 1977; Ribak et al., 2000; Shapiro and Ribak, 2006).

Activation of astrocytes is another hallmark feature of OP intoxication (Zimmer et al., 1997; Chen, 2012; Rojas et al., 2015; Sisó et al., 2017). Astrocytes, which account for 20\%$40 \%$ of all glial cells in the brain, provide metabolic support for neurons and help in the maintenance of the BBB integrity. Our experiments with GFAP and IBA1 staining indicated significant changes in morphology and proliferation of astrocytes and microglial cells in many brain regions after DPF exposure. DFP-triggered cellular neuroinflammation was effectively prevented by early administration of MDZ. The up-regulations of microglia and astrocytes are prominent in delayed MDZ groups (40 minutes and later). The extent of microglia and astrocyte activation is strongly correlated with elapsed time for MDZ treatment (40-120 minutes) after exposure to DFP that caused seizures and SE within 10 minutes (Figs. 2 and 10). Protracted cellular neuroinflammatory response after OP intoxication may ultimately contribute to long-term structural and neuropsychiatric dysfunction, as observed in Tokyo victims exposed to sarin (Yamasue et al., 2007).

Benzodiazepines are the drugs of choice for treatment of SE because they are easy to use, enter the brain rapidly, elicit a quick onset of action, possess an optimal half-life, and are available in multiple formulations (Goodkin and Kapur, 2009). MDZ has been proposed to replace diazepam in nerve agent treatment kits. MDZ has several advantages for SE treatment, including rapid onset of action, short half-life, water solubility, and longer shelf-life in an injectable formulation (Reddy and Reddy, 2015). MDZ has been tested in a variety of animal models of SE (McDonough et al., 1999; Shih et al., 1999; Capacio et al., 2004; RamaRao et al., 2014). Among benzodiazepines, MDZ or MDZ combined with an anticholinergic drug was found to be the most effective for terminating soman-induced seizures when given early after seizure onset in guinea pig models (McDonough et al., 1999; Koplovitz et al., 2001). In the RAMPART clinical trial, administration of MDZ before arrival to the emergency room had favorable SE termination rates (Silbergleit et al., 2012). The overall efficacy of benzodiazepines in SE depends on time of administration after seizure onset (Alldredge et al., 2001; Silbergleit et al., 2012).

The mechanisms underlying the development of resistance to benzodiazepines remain unclear. To uncover these potential cellular and molecular mechanisms, both electrical and chemical models of SE exhibiting resistance to benzodiazepines were used (Mazarati et al., 1998; McDonough et al., 2010; Apland et al., 2014; Kuruba et al., 2018; Lewczuk et al., 2018). Benzodiazepine resistance may occur due to rapid changes in GABAergic inhibition and/or increase in glutamatergic excitation (Goodkin et al., 2005; Naylor et al., 2005; Joshi et al., 2017). Reduced benzodiazepine efficacy in SE can arise from multiple mechanisms including trafficking or internalization of synaptic GABA-A receptors or other dysfunctions in GABAergic synaptic transmission leading to diminished inhibition ( 50\%) in neuronal circuits (Goodkin et al., 2003, 2005, 2007, 2008; Naylor et al., 2005; Deeb et al., 2012; Vinkers and Olivier, 2012). Concerted changes that occurs with persistent seizuressuch as trafficking or down-regulation of synaptic GABA-A receptors, up-regulation of glutamate ( $N$-methyl-D-aspartate [NMDA]/ $\alpha$-amino-3-hydroxy-5-methyl-4-isoxazole propionic acid [AMPA]) receptors in the dentate gyrus, and compromise in BBB permeability —an lead to refractory or self-sustaining SE. These changes may also account for the apparent MDZ pharmacoresistance often secondary to a lack of available postsynaptic GABA-A receptors (Goodkin and Kapur, 2009; Qashu et al., 2010; Naylor et al., 2013; Joshi et al., 2017; Carver et al., 2014; Carver and Reddy, 2016; Reddy et al., 2015; Wu et al., 2013). Moreover, MDZ has a short half-life ( 2 hours), so it needs repeated injections or to be combined with other adjunct anticonvulsants. It is likely that repeated administration or high doses of MDZ may result in sedation, cardiovascular and respiratory depression, and tolerance (Reddy and Reddy, 2015; Holtkamp, 2018).

In conclusion, these results are consistent with prior reports (Deshpande et al., 2010; Pouliot et al., 2016; Sisó et al., 2017) demonstrating that the rat model of acute DFP intoxication recapitulates many features of acute exposure to OP nerve agents, including persistent SE, neurodegeneration, and neuroinflammatory responses. This study demonstrates that MDZ is an effective anticonvulsant for controlling DFP-induced $\mathrm{SE}$ when given within 10 minutes. However, these seizures are progressively resistant to MDZ if treatment is delayed (40 minutes or later), indicating benzodiazepine refractory SE. Late MDZ therapy (40 minutes or later) is associated with significantly severe brain damage, which could lead to the long-term neurologic dysfunction of acute OP intoxication. Thus, there is an urgent need to develop novel anticonvulsants and neuroprotectants superior to MDZ or adjunct drugs that can enhance its efficacy for effective treatment of SE and neuronal injury after OP intoxication.

\section{Acknowledgments}

We thank Victoria Golub, Dheepthi Perumal, and Jenessa Short for technical help with stereology counts and Dr. Suojin Wang for advice on the statistical analysis.

\section{Authorship Contributions}

Participated in research design: Reddy.

Conducted experiments: $\mathrm{Wu}$, Kuruba, Reddy.

Performed data analysis: Wu, Kuruba, Reddy.

Wrote or contributed to the writing of the manuscript: Wu, Reddy.

\section{References}

Abou-Donia MB, Siracuse B, Gupta N, and Sobel Sokol A (2016) Sarin (GB, O-isopropyl methylphosphonofluoridate) neurotoxicity: critical review. Crit Rev Toxicol 46:845-875.

Alldredge BK, Gelb AM, Isaacs SM, Corry MD, Allen F, Ulrich S, Gottwald MD, O'Neil N, Neuhaus JM, Segal MR, et al. (2001) A comparison of lorazepam, diazepam, and placebo for the treatment of out-of-hospital status epilepticus. $N$ Engl J Med 345:631-637.

André V, Marescaux C, Nehlig A, and Fritschy JM (2001) Alterations of hippocampal GAbaergic system contribute to development of spontaneous recurrent seizures in the rat lithium-pilocarpine model of temporal lobe epilepsy. Hippocampus 11: $452-468$.

Apland JP, Aroniadou-Anderjaska V, Figueiredo TH, Rossetti F, Miller SL, and Braga MF (2014) The limitations of diazepam as a treatment for nerve agent-induced seizures and neuropathology in rats: comparison with UBP302. J Pharmacol Exp Ther 351:359-372. Apland JP, Figueiredo TH, Qashu F, Aroniadou-Anderjaska V, Souza AP, and Braga MF (2010) Higher susceptibility of the ventral versus the dorsal hippocampus and the posteroventral versus anterodorsal amygdala to soman-induced neuropathology. Neurotoxicology 31:485-492.

Aroniadou-Anderjaska V, Figueiredo TH, Apland JP, Qashu F, and Braga MF (2009) Primary brain targets of nerve agents: the role of the amygdala in comparison to the hippocampus. Neurotoxicology 30:772-776.

Baille-Le Crom V, Collombet JM, Carpentier P, Brochier G, Burckhart MF, Foquin A, Pernot-Marino I, Rondouin G, and Lallement G (1995) Early regional changes of GFAP mRNA in rat hippocampus and dentate gyrus during soman-induced seizures. Neuroreport 7:365-369.

Bajgar J (1997) Differential inhibition of the brain acetylcholinesterase molecular forms following sarin, soman and VX intoxication in laboratory rats. Acta Med (Hradec Kralove) 40:89-94.

Bajgar J (2004) Organophosphates/nerve agent poisoning: mechanism of action, diagnosis, prophylaxis, and treatment. Adv Clin Chem 38:151-216. 
Bajgar J, Fusek J, Kassa J, Jun D, Kuca K, and Hájek P (2008) An attempt to assess functionally minimal acetylcholinesterase activity necessary for survival of rats intoxicated with nerve agents. Chem Biol Interact 175:281-285.

Bakry NM, el-Rashidy AH, Eldefrawi AT, and Eldefrawi ME (1988) Direct actions of organophosphate anticholinesterases on nicotinic and muscarinic acetylcholine receptors. J Biochem Toxicol 3:235-259.

Banks CN and Lein PJ (2012) A review of experimental evidence linking neurotoxic organophosphorus compounds and inflammation. Neurotoxicology 33:575-584.

Boccia MM, Blake MG, Acosta GB, and Baratti CM (2003) Atropine, an anticholinergic drug, impairs memory retrieval of a high consolidated avoidance response in mice. Neurosci Lett 345:97-100.

Bouzarth WF and Himwich HE (1952) Mechanism of seizures induced by di-isopropyl fluorophosphate. Am J Psychiatry 108:847-855.

Boyce RW, Dorph-Petersen KA, Lyck L, and Gundersen HJ (2010) Design-based stereology: introduction to basic concepts and practical approaches for estimation of cell number. Toxicol Pathol 38:1011-1025.

Briyal S and Reddy DS (2008) Neuroactive steroid therapy of status epilepticus in epilepsy rats. Epilepsia 49 (Suppl 7):3.055

Capacio BR, Byers CE, Merk KA, Smith JR, and McDonough JH (2004) Pharmacokinetic studies of intramuscular midazolam in guinea pigs challenged with soman. Drug Chem Toxicol 27:95-110.

Capacio BR, Whalley CE, Byers CE, and McDonough JH (2001) Intramuscular diazepam pharmacokinetics in soman-exposed guinea pigs. J Appl Toxicol 21 (Suppl 1):S67-S74.

Carver CM and Reddy DS (2016) Neurosteroid structure-activity relationships for functional activation of extrasynaptic $\delta$ GABA(A) receptors. J Pharmacol Exp Ther 357:188-204.

Carver CM, Wu X, Gangisetty O, and Reddy DS (2014) Perimenstrual-like hormonal regulation of extrasynaptic $\delta$-containing GABAA receptors mediating tonic inhibition and neurosteroid sensitivity. $J$ Neurosci 34:14181-14197.

Chapman S, Yaakov G, Egoz I, Rabinovitz I, Raveh L, Kadar T, Gilat E, and Grauer $\mathrm{E}$ (2015) Sarin-induced brain damage in rats is attenuated by delayed administration of midazolam. Neurotoxicology 49:132-138.

Chen J, Pan H, Chen C, Wu W, Iskandar K, He J, Piermartiri T, Jacobowitz DM, Yu QS, McDonough JH, et al. (2014) (-)-Phenserine attenuates soman-induced neuropathology. PLoS One 9(6):e99818.

Chen Y (2012) Organophosphate-induced brain damage: mechanisms, neuropsychiatric and neurological consequences, and potential therapeutic strategies. Neurotoxicology 33:391-400.

Collombet JM, Four E, Bernabé D, Masqueliez C, Burckhart MF, Baille V, Baubichon D, and Lallement G (2005) Soman poisoning increases neural progenitor proliferation and induces long-term glial activation in mouse brain. Toxicology 208:319-334.

de Araujo Furtado M, Lumley LA, Robison C, Tong LC, Lichtenstein S, and Yourick DL (2010) Spontaneous recurrent seizures after status epilepticus induced by soman in Sprague-Dawley rats. Epilepsia 51:1503-1510.

Deeb TZ, Maguire J, and Moss SJ (2012) Possible alterations in GABAA receptor signaling that underlie benzodiazepine-resistant seizures. Epilepsia 53 (Suppl 9): $79-88$.

Deshpande LS, Carter DS, Blair RE, and DeLorenzo RJ (2010) Development of a prolonged calcium plateau in hippocampal neurons in rats surviving status epilepticus induced by the organophosphate diisopropylfluorophosphate. Toxicol Sci 116:623-631.

Dolgin E (2013) Syrian gas attack reinforces need for better anti-sarin drugs. Nat Med 19:1194-1195.

Dorph-Petersen KA and Lewis DA (2011) Stereological approaches to identifying neuropathology in psychosis. Biol Psychiatry 69:113-126.

Eddleston M, Buckley NA, Eyer P, and Dawson AH (2008) Management of acute organophosphorus pesticide poisoning. Lancet 371:597-607.

Ferchmin PA, Andino M, Reyes Salaman R, Alves J, Velez-Roman J, Cuadrado B, Carrasco M, Torres-Rivera W, Segarra A, Martins AH, et al. (2014) 4R-cembranoid protects against diisopropylfluorophosphate-mediated neurodegeneration. Neurotoxicology 44:80-90.

Fernandez A, Lantigua H, Lesch C, Shao B, Foreman B, Schmidt JM, Hirsch LJ, Mayer SA, and Claassen J (2014) High-dose midazolam infusion for refractory status epilepticus. Neurology 82:359-365.

Figueiredo TH, Qashu F, Apland JP, Aroniadou-Anderjaska V, Souza AP, and Braga MF (2011) The GluK1 (GluR5) Kainate/\{alpha\}-amino-3-hydroxy-5-methyl-4isoxazolepropionic acid receptor antagonist LY293558 reduces soman-induced seizures and neuropathology. J Pharmacol Exp Ther 336:303-312.

Flannery BM, Bruun DA, Rowland DJ, Banks CN, Austin AT, Kukis DL, Li Y, Ford BD, Tancredi DJ, Silverman JL, et al. (2016) Persistent neuroinflammation and cognitive impairment in a rat model of acute diisopropylfluorophosphate intoxication. $J$ Neuroinflammation 13:267.

Fujikawa DG (1996) The temporal evolution of neuronal damage from pilocarpineinduced status epilepticus. Brain Res 725:11-22.

Gilbert DL, Gartside PS, and Glauser TA (1999) Efficacy and mortality in treatment of refractory generalized convulsive status epilepticus in children: a meta-analysis. $J$ Child Neurol 14:602-609.

Glauser T, Shinnar S, Gloss D, Alldredge B, Arya R, Bainbridge J, Bare M, Bleck T, Dodson WE, Garrity L, et al. (2016) Evidence-based guideline: treatment of convulsive status epilepticus in children and adults: report of the Guideline Committee of the American Epilepsy Society. Epilepsy Curr 16:48-61.

Golub VM, Brewer J, Wu X, Kuruba R, Short J, Manchi M, Swonke M, Younus I, and Reddy DS (2015) Neurostereology protocol for unbiased quantification of neuronal injury and neurodegeneration. Front Aging Neurosci 7:196.

Goodkin HP, Joshi S, Mtchedlishvili Z, Brar J, and Kapur J (2008) Subunit-specific trafficking of GABA(A) receptors during status epilepticus. $J$ Neurosci 28 $2527-2538$

Goodkin HP and Kapur J (2009) The impact of diazepam's discovery on the treatment and understanding of status epilepticus. Epilepsia 50:2011-2018.
Goodkin HP, Liu X, and Holmes GL (2003) Diazepam terminates brief but not prolonged seizures in young, naïve rats. Epilepsia 44:1109-1112.

Goodkin HP, Sun C, Yeh JL, Mangan PS, and Kapur J (2007) GABA(A) receptor internalization during seizures. Epilepsia 48 (Suppl 5):109-113.

Goodkin HP, Yeh JL, and Kapur J (2005) Status epilepticus increases the intracellular accumulation of GABAA receptors. J Neurosci 25:5511-5520.

Gruber B, Greber S, Rupp E, and Sperk G (1994) Differential NPY mRNA expression in granule cells and interneurons of the rat dentate gyrus after kainic acid injection. Hippocampus 4:474-482.

Gunnell D and Eddleston M (2003) Suicide by intentional ingestion of pesticides: a continuing tragedy in developing countries. Int $J$ Epidemiol 32:902-909.

Hájek P, Slízová D, Krs O, and Bajgar J (2004) Comparison of changes in AChE activity in the brain of the laboratory rat after soman and tabun intoxication. Biomed Pap Med Fac Univ Palacky Olomouc Czech Repub 148:209-211.

Hassel B (2006) Nicotinic mechanisms contribute to soman-induced symptoms and lethality. Neurotoxicology 27:501-507.

Hattiangady B, Kuruba R, and Shetty AK (2011) Acute seizures in old age leads to a greater loss of CA1 pyramidal neurons, an increased propensity for developing chronic TLE and a severe cognitive dysfunction. Aging Dis 2:1-17.

Hayward IJ, Wall HG, Jaax NK, Wade JV, Marlow DD, and Nold JB (1990) Decreased brain pathology in organophosphate-exposed rhesus monkeys following benzodiazepine therapy. J Neurol Sci 98:99-106.

Heiss DR, Zehnder DW, II, Jett DA, Platoff GE Jr, Yeung DT, and Brewer BN (2016 Synthesis and storage stability of diisopropylfluorophosphate. J Chem 2016: 3190891.

Hobson BA, Rowland DJ, Supasai S, Harvey DJ, Lein PJ, and Garbow JR (2018) A magnetic resonance imaging study of early brain injury in a rat model of acute DFP intoxication. Neurotoxicology 66:170-178

Hobson BA, Sisó S, Rowland DJ, Harvey DJ, Bruun DA, Garbow JR, and Lein PJ (2017) From the cover: magnetic resonance imaging reveals progressive brain injury in rats acutely intoxicated with diisopropylfluorophosphate. Toxicol Sci 157:342-353.

Holtkamp M (2018) Pharmacotherapy for refractory and super-refractory status epilepticus in adults. Drugs 78:307-326.

Jett DA and Yeung DT (2010) The CounterACT Research Network: basic mechanisms and practical applications. Proc Am Thorac Soc 7:254-256.

Jokanović M and Kosanović M (2010) Neurotoxic effects in patients poisoned with organophosphorus pesticides. Environ Toxicol Pharmacol 29:195-201.

Joshi S, Rajasekaran K, Sun H, Williamson J, and Kapur J (2017) Enhanced AMPA receptor-mediated neurotransmission on CA1 pyramidal neurons during status epilepticus. Neurobiol Dis 103:45-53.

Kadriu B, Guidotti A, Costa E, and Auta J (2009) Imidazenil, a non-sedating anticonvulsant benzodiazepine, is more potent than diazepam in protecting against DFP-induced seizures and neuronal damage. Toxicology 256:164-174.

Kadriu B, Guidotti A, Costa E, Davis JM, and Auta J (2011) Acute imidazenil treatment after the onset of DFP-induced seizure is more effective and longer lasting than midazolam at preventing seizure activity and brain neuropathology. Toxicol Sci 120:136-145.

Kenley RA, Howd RA, and Uyeno ET (1982) Effects of PAM, proPAM, and DFP on behavior, thermoregulation, and brain AChE in rats. Pharmacol Biochem Behav 17:1001-1008.

Kim YB, Hur GH, Shin S, Sok DE, Kang JK, and Lee YS (1999) Organophosphateinduced brain injuries: delayed apoptosis mediated by nitric oxide. Environ Toxicol Pharmacol 7:147-152.

Kobayashi M and Buckmaster PS (2003) Reduced inhibition of dentate granule cells in a model of temporal lobe epilepsy. J Neurosci 23:2440-2452.

Koplovitz I, Schulz S, Shutz M, Railer R, Macalalag R, Schons M, and McDonough J (2001) Combination anticonvulsant treatment of soman-induced seizures. J Appl Toxicol 21 (Suppl 1):S53-S55.

Krause KH, van Thriel C, De Sousa PA, Leist M, and Hengstler JG (2013) Monocrotophos in Gandaman village: India school lunch deaths and need for improved toxicity testing. Arch Toxicol 87:1877-1881.

Kuruba R, Hattiangady B, Parihar VK, Shuai B, and Shetty AK (2011) Differential susceptibility of interneurons expressing neuropeptide Y or parvalbumin in the aged hippocampus to acute seizure activity. PLoS One 6:e24493.

Kuruba R, Wu X, and Reddy DS (2018) Benzodiazepine-refractory status epilepticus, neuroinflammation, and interneuron neurodegeneration after acute organophosphate intoxication. Biochim Biophys Acta 1864:2845-2858.

Kwong TC (2002) Organophosphate pesticides: biochemistry and clinical toxicology. Ther Drug Monit 24:144-149.

Lallement G, Clarencon D, Brochier G, Baubichon D, Galonnier M, Blanchet G, and Mestries JC (1997) Efficacy of atropine/pralidoxime/diazepam or atropine/HI6/prodiazepam in primates intoxicated by soman. Pharmacol Biochem Behav 56: 325-332.

Lee J, Huh L, and Korn P (2011) Guideline for the management of convulsive status epilepticus in infants and children. BC Med J 53:279-285.

Leikin JB, Thomas RG, Walter FG, Klein R, and Meislin HW (2002) A review of nerve agent exposure for the critical care physician. Crit Care Med 30:2346-2354.

Lemos T and Cavalheiro EA (1995) Suppression of pilocarpine-induced status epilepticus and the late development of epilepsy in rats. Exp Brain Res 102:423-428.

Lewczuk E, Joshi S, Williamson J, Penmetsa M, Shan S, and Kapur J (2018) Electroencephalography and behavior patterns during experimental status epilepticus. Epilepsia 59:369-380.

Li Y, Lein PJ, Ford GD, Liu C, Stovall KC, White TE, Bruun DA, Tewolde T, Gates AS, Distel TJ, et al. (2015) Neuregulin-1 inhibits neuroinflammatory responses in a rat model of organophosphate-nerve agent-induced delayed neuronal injury. $J$ Neuroinflammation 12:64.

Li Y, Lein PJ, Liu C, Bruun DA, Tewolde T, Ford G, and Ford BD (2011) Spatiotemporal pattern of neuronal injury induced by DFP in rats: a model for delayed neuronal cell death following acute OP intoxication. Toxicol Appl Pharmacol 253. 261-269. 
Liang LP, Pearson-Smith JN, Huang J, McElroy P, Day BJ, and Patel M (2018) Neuroprotective effects of AEOL10150 in a rat organophosphate model. Toxicol Sci 162:611-621.

Liu C, Li Y, Lein PJ, and Ford BD (2012) Spatiotemporal patterns of GFAP upregulation in rat brain following acute intoxication with diisopropylfluorophosphate (DFP). Curr Neurobiol 3:90-97.

Mazarati AM, Wasterlain CG, Sankar R, and Shin D (1998) Self-sustaining status epilepticus after brief electrical stimulation of the perforant path. Brain Res 801: 251-253.

McDonough JH Jr, Dochterman LW, Smith CD, and Shih TM (1995) Protection against nerve agent-induced neuropathology, but not cardiac pathology, is associated with the anticonvulsant action of drug treatment. Neurotoxicology $\mathbf{1 6}$ 123-132.

McDonough JH Jr, McMonagle J, Copeland T, Zoeffel D, and Shih TM (1999) Comparative evaluation of benzodiazepines for control of soman-induced seizures. Arch Toxicol 73:473-478.

McDonough JH, McMonagle JD, and Shih TM (2010) Time-dependent reduction in the anticonvulsant effectiveness of diazepam against soman-induced seizures in Guinea pigs. Drug Chem Toxicol 33:279-283.

McDonough JH Jr and Shih TM (1997) Neuropharmacological mechanisms of nerve agent-induced seizure and neuropathology. Neurosci Biobehav Rev 21:559-579.

McDonough JH, Van Shura KE, LaMont JC, McMonagle JD, and Shih TM (2009) Comparison of the intramuscular, intranasal or sublingual routes of midazolam administration for the control of soman-induced seizures. Basic Clin Pharmacol Toxicol 104:27-34.

McDonough JH Jr, Zoeffel LD, McMonagle J, Copeland TL, Smith CD, and Shih TM (2000) Anticonvulsant treatment of nerve agent seizures: anticholinergics versus diazepam in soman-intoxicated Guinea pigs. Epilepsy Res 38:1-14.

McElroy PB, Liang LP, Day BJ, and Patel M (2017) Scavenging reactive oxygen species inhibits status epilepticus-induced neuroinflammation. Exp Neurol $\mathbf{2 9 8}$ (Pt A): $13-22$

Mullen RJ, Buck CR, and Smith AM (1992) NeuN, a neuronal specific nuclear protein in vertebrates. Development 116:201-211.

Myhrer T, Andersen JM, Nguyen NH, and Aas P (2005) Soman-induced convulsions in rats terminated with pharmacological agents after $45 \mathrm{~min}$ : neuropathology and cognitive performance. Neurotoxicology 26:39-48.

Naylor DE, Liu H, and Wasterlain CG (2005) Trafficking of GABA(A) receptors, loss of inhibition, and a mechanism for pharmacoresistance in status epilepticus. $J$ Neurosci 25:7724-7733.

Naylor DE, Liu H, Niquet J, and Wasterlain CG (2013) Rapid surface accumulation of NMDA receptors increases glutamatergic excitation during status epilepticus Neurobiol Dis 54:225-238.

Paxinos G and Watson C (2007) The Rat Brain in Stereotaxic Coordinates, 6th ed, Academic Press, New York.

Pereira EF, Aracava Y, DeTolla LJ Jr, Beecham EJ, Basinger GW Jr, Wakayama EJ, and Albuquerque EX (2014) Animal models that best reproduce the clinical manifestations of human intoxication with organophosphorus compounds. J Pharmacol Exp Ther 350:313-321.

Pessah IN, Rogawski MA, Tancredi DJ, Wulff H, Zolkowska D, Bruun DA, Hammock BD, and Lein PJ (2016) Models to identify treatments for the acute and persistent effects of seizure-inducing chemical threat agents. Ann N Y Acad Sci 1378:124-136.

Petito CK, Schaefer JA, and Plum F (1977) Ultrastructural characteristics of the brain and blood-brain barrier in experimental seizures. Brain Res 127: 251-267.

Pittel Z, Barak D, and Segall Y (2006) Function-specific blockage of M(1) and M(3) muscarinic acetylcholine receptors by VX and echothiophate. Brain Res $\mathbf{1 0 8 5}$ 102-110.

Pittel Z, Lazar S, Gez R, and Chapman S (2018) Early changes in M2 muscarinic acetylcholine receptors (mAChRs) induced by sarin intoxication may be linked to long lasting neurological effects. Neurotoxicology 65:248-254.

Pouliot W, Bealer SL, Roach B, and Dudek FE (2016) A rodent model of human organophosphate exposure producing status epilepticus and neuropathology. Neurotoxicology 56:196-203.

Prager EM, Aroniadou-Anderjaska V, Almeida-Suhett CP, Figueiredo TH, Apland JP, and Braga MF (2013) Acetylcholinesterase inhibition in the basolateral amygdala plays a key role in the induction of status epilepticus after soman exposure. Neurotoxicology 38:84-90.

Prager EM, Aroniadou-Anderjaska V, Almeida-Suhett CP, Figueiredo TH, Apland JP, Rossetti F, Olsen CH, and Braga MF (2014) The recovery of acetylcholinesterase activity and the progression of neuropathological and pathophysiological alterations in the rat basolateral amygdala after soman-induced status epilepticus: relation to anxiety-like behavior. Neuropharmacology 81:64-74

Qashu F, Figueiredo TH, Aroniadou-Anderjaska V, Apland JP, and Braga MF (2010) Diazepam administration after prolonged status epilepticus reduces neurodegeneration in the amygdala but not in the hippocampus during epileptogenesis. Amino Acids 38:189-197.

Racine RJ (1972) Modification of seizure activity by electrical stimulation. II. Motor seizure. Electroencephalogr Clin Neurophysiol 32:281-294.

RamaRao G, Afley P, Acharya J, and Bhattacharya BK (2014) Efficacy of antidotes (midazolam, atropine and HI-6) on nerve agent induced molecular and neuropathological changes. BMC Neurosci 15:47.

Rao MS, Hattiangady B, Reddy DS, and Shetty AK (2006) Hippocampal neurodegeneration, spontaneous seizures, and mossy fiber sprouting in the F344 rat model of temporal lobe epilepsy. J Neurosci Res 83:1088-1105.

Reddy DS (2014) Clinical pharmacology of current antiepileptic drugs. Int $J$ Pharma Sci Nanotech 7:2305-2319.

Reddy DS (2016) Neurosteroids for the potential protection of humans against organophosphate toxicity. Ann N Y Acad Sci 1378:25-32.
Reddy DS and Colman E (2017) A comparative toxidrome analysis of human organophosphate and nerve agent poisonings using social media. Clin Transl Sci 10 $225-230$.

Reddy DS and Kuruba R (2013) Experimental models of status epilepticus and neuronal injury for evaluation of therapeutic interventions. Int J Mol Sci 14 18284-18318.

Reddy SD and Reddy DS (2015) Midazolam as an anticonvulsant antidote for organophosphate intoxication-a pharmacotherapeutic appraisal. Epilepsia 56: 813-821.

Reddy SD, Younus I, Clossen BL, and Reddy DS (2015) Antiseizure activity of midazolam in mice lacking $\delta$-subunit extrasynaptic GABA(A) receptors. $J$ Pharmacol Exp Ther 353:517-528.

Ribak CE, Tran PH, Spigelman I, Okazaki MM, and Nadler JV (2000) Status epilepticus-induced hilar basal dendrites on rodent granule cells contribute to recurrent excitatory circuitry. $J$ Comp Neurol 428:240-253.

Rogin J, Wheless J, Abou-Khalil B, Wolter KD, Pixton GC, Sherman NA, Shukla RB, Roland CL, and Sommerville KW (2014) Safety and effectiveness of longterm treatment with diazepam auto-injector administered by caregivers in an outpatient setting for the treatment of acute repetitive seizures. Epilepsia $\mathbf{5 5}$ : $1444-1451$.

Rojas A, Ganesh T, Lelutiu N, Gueorguieva P, and Dingledine R (2015) Inhibition of the prostaglandin EP2 receptor is neuroprotective and accelerates functional recovery in a rat model of organophosphorus induced status epilepticus. Neuropharmacology 93:15-27.

Rojas A, Wang W, Glover A, Manji Z, Fu Y, and Dingledine R (2018) Beneficial outcome of urethane treatment following status epilepticus in a rat organophosphorus toxicity model. eNeuro 5(2):ENEURO.0070-18.2018.

Rosman Y, Eisenkraft A, Milk N, Shiyovich A, Ophir N, Shrot S, Kreiss Y, and Kassirer M (2014) Lessons learned from the Syrian sarin attack: evaluation of a clinical syndrome through social media. Ann Intern Med 160: $644-648$

Sakurada K, Matsubara K, Shimizu K, Shiono H, Seto Y, Tsuge K, Yoshino M, Sakai I, Mukoyama H, and Takatori T (2003) Pralidoxime iodide (2-pAM) penetrates across the blood-brain barrier. Neurochem Res 28:1401-1407.

Savage EP, Keefe TJ, Mounce LM, Heaton RK, Lewis JA, and Burcar PJ (1988) Chronic neurological sequelae of acute organophosphate pesticide poisoning. Arch Environ Health 43:38-45.

Schmued LC, Albertson C, and Slikker W Jr (1997) Fluoro-Jade: a novel fluorochrome for the sensitive and reliable histochemical localization of neuronal degeneration. Brain Res 751:37-46.

Scholl EA, Miller-Smith SM, Bealer SL, Lehmkuhle MJ, Ekstrand JJ, Dudek FE, and McDonough JH (2018) Age-dependent behaviors, seizure severity and neuronal damage in response to nerve agents or the organophosphate DFP in immature and adult rats. Neurotoxicology 66:10-21.

Shapiro LA and Ribak CE (2006) Newly born dentate granule neurons after pilocarpine-induced epilepsy have hilar basal dendrites with immature synapses. Epilepsy Res 69:53-66.

Shetty AK, Rao MS, Hattiangady B, Zaman V, and Shetty GA (2004) Hippocampal neurotrophin levels after injury: relationship to the age of the hippocampus at the time of injury. J Neurosci Res 78:520-532.

Shih T, McDonough JH Jr, and Koplovitz I (1999) Anticonvulsants for soman-induced seizure activity. J Biomed $S c i$ 6:86-96.

Shih TM, Duniho SM, and McDonough JH (2003) Control of nerve agent-induced seizures is critical for neuroprotection and survival. Toxicol Appl Pharmacol 188: 69-80.

Shih TM, Koviak TA, and Capacio BR (1991) Anticonvulsants for poisoning by the organophosphorus compound soman: pharmacological mechanisms. Neurosci Biobehav Rev 15:349-362.

Shih TM and McDonough JH Jr (1999) Organophosphorus nerve agents-induced seizures and efficacy of atropine sulfate as anticonvulsant treatment. Pharmaco Biochem Behav 64:147-153.

Shih TM, Skovira JW, and McDonough JH (2009) Effects of 4-pyridine aldoxime on nerve agent-inhibited acetylcholinesterase activity in Guinea pigs. Arch Toxicol $\mathbf{8 3}$ 1083-1089.

Shih TM, Skovira JW, O'Donnell JC, and McDonough JH (2010) Treatment with tertiary oximes prevents seizures and improves survival following sarin intoxication. J Mol Neurosci 40:63-69.

Silbergleit R, Durkalski V, Lowenstein D, Conwit R, Pancioli A, Palesch Y, and Barsan W; NETT Investigators (2012) Intramuscular versus in travenous therapy for prehospital status epilepticus. $N$ Engl J Med 366: $591-600$

Silbergleit R, Lowenstein D, Durkalski V, and Conwit R; Neurological Emergency Treatment Trials (NETT) Investigators (2011) RAMPART (Rapid Anticonvulsant Medication Prior to Arrival Trial): a double-blind randomized clinical trial of the efficacy of intramuscular midazolam versus intravenous lorazepam in the prehospital treatment of status epilepticus by paramedics. Epilepsia $\mathbf{5 2}$ (Suppl 8): $45-47$.

Sirin GS, Zhou Y, Lior-Hoffmann L, Wang S, and Zhang Y (2012) Aging mechanism of soman inhibited acetylcholinesterase. J Phys Chem B 116:12199-12207.

Sisó S, Hobson BA, Harvey DJ, Bruun DA, Rowland DJ, Garbow JR, and Lein PJ (2017) Editor's highlight: Spatiotemporal progression and remission of lesions in the rat brain following acute intoxication with diisopropylfluorophosphate. Toxicol Sci 157:330-341.

Skovira JW, McDonough JH, and Shih TM (2010) Protection against sarin-induced seizures in rats by direct brain microinjection of scopolamine, midazolam or MK-801. J Mol Neurosci 40:56-62.

Smith R and Brown J (2017) Midazolam for status epilepticus. Aust Prescr 40:23-25. Stroup WW (2012) Generalized Linear Mixed Models: Modern Concepts, Methods and Applications, CRC Press, Boca Raton, FL. 
Vinkers CH and Olivier B (2012) Mechanisms underlying tolerance after long-term benzodiazepine use: a future for subtype-selective GABA(A) receptor modulators? Adv Pharmacol Sci 2012:416864.

Wright LK, Liu J, Nallapaneni A, and Pope CN (2010) Behavioral sequelae following acute diisopropylfluorophosphate intoxication in rats: comparative effects of atropine and cannabinomimetics. Neurotoxicol Teratol 32 329-335.

Wu X, Gangisetty O, Carver CM, and Reddy DS (2013) Estrous cycle regulation of extrasynaptic $\delta$-containing GABA(A) receptor plasticity and tonic inhibition in the hippocampus subfields. J Pharmacol Exp Ther 346:146-160.

Yamasue H, Abe O, Kasai K, Suga M, Iwanami A, Yamada H, Tochigi M, Ohtani T, Rogers MA, Sasaki T, et al. (2007) Human brain structural change related to acute single exposure to sarin. Ann Neurol 61:37-46.
Yanagisawa N, Morita H, and Nakajima T (2006) Sarin experiences in Japan: acute toxicity and long-term effects. J Neurol Sci 249:76-85.

Yokoyama K (2007) Our recent experiences with sarin poisoning cases in Japan and pesticide users with references to some selected chemicals. Neurotoxicology 28:364-373. Zimmer LA, Ennis M, and Shipley MT (1997) Soman-induced seizures rapidly activate astrocytes and microglia in discrete brain regions. J Comp Neurol 378:482-492.

Address correspondence to: Dr. Doodipala Samba Reddy, Department of Neuroscience and Experimental Therapeutics, Texas A\&M University Health Science Center College of Medicine, 8447 Riverside Pkwy, MREB Building 2008, Bryan, TX 77807-3260. E-mail: reddy@medicine.tamhsc.edu 